\title{
A HIGH RESOLUTION STUDY OF THE HYDRA A CLUSTER WITH CHANDRA: COMPARISON OF THE CORE MASS DISTRIBUTION WITH THEORETICAL PREDICTIONS AND EVIDENCE FOR FEEDBACK IN THE COOLING FLOW.
}

\author{
L.P. David,${ }^{1}$ P.E.J. Nulsen, ${ }^{2}$ B.R. McNamara, ${ }^{1,3}$ W. Forman,${ }^{1}$ C. Jones,${ }^{1}$ T. Ponman,${ }^{4}$, \\ B. RobERTSON, ${ }^{5}$ AND M. Wise, ${ }^{6}$
}

accepted for publication in the The Astrophysical Journal

\begin{abstract}
The cooling flow cluster Hydra A was observed during the orbital activation and calibration phase of the Chandra Observatory. While the X-ray image of the cluster exhibits complex structure in the central region as reported in McNamara etal, the large scale X-ray morphology of the cluster is fairly smooth. A spectroscopic analysis of the ACIS data shows that the gas temperature in Hydra A increases outward, reaches a maximum temperature of $4 \mathrm{keV}$ at $200 \mathrm{kpc}$, and then decreases slightly at larger radii. The distribution of heavy elements is nonuniform, with a factor of two increase in the $\mathrm{Fe}$ and $\mathrm{Si}$ abundances within the central $100 \mathrm{kpc}$. Beyond the central $100 \mathrm{kpc}$ the $\mathrm{Si}$-to-Fe abundance ratio is twice solar, while the Si-to-Fe ratio of the central excess is consistent with the solar value. One of the more surprising results is the lack of spectroscopic evidence for multiphase gas within the bulk of the cooling flow. Beyond the central $30 \mathrm{kpc}$, the ACIS spectra are adequately fit with a single temperature model. The addition of a cooling flow component does not significantly improve the fit. Only within the central $30 \mathrm{kpc}$ (where the cooling time is less than $1 \mathrm{Gyr}$ ), is there spectroscopic evidence for multiphase gas. However, the spectroscopic mass deposition rate is more than a factor of 10 less than the morphologically derived mass accretion rate at $30 \mathrm{kpc}$. We propose that the cooling flow region is convectively unstable due to heating by the central radio source which significantly reduces the net accretion rate. In addition, we show that the mass distribution within the central 30-200 kpc region scales as $\rho_{d} \propto r^{-1.3}$, intermediate between an NFW and Moore profile, but with a best-fit NFW concentration parameter $\left(c_{N F W}=12\right)$ approximately 3 times greater than that found in numerical simulations. However, given the limited photon statistics, we cannot rule out the presence of a flat-density core with a core radius less than $30 \mathrm{kpc}$.
\end{abstract}

Subject headings: cooling flows-galaxies:clusters:individual Hydra A

\section{INTRODUCTION}

The launch of the Chandra X-ray Observatory (Weisskopf et al. 2000) provides astronomers with the first opportunity to view clusters of galaxies with the same spatial resolution as ground based optical telescopes. This improved resolution is especially beneficial in the study of cooling flow clusters in which most of the interesting astrophysics is confined to the central 100 kpc (Fabian \& Nulsen 1977; Sarazin 1986; Fabian 1994). One of the great mysteries concerning cooling flows has always been the contrasting implications of the X-ray observations with observations at other wavelengths. While Xray observations suggest that a substantial amount of hot gas (up to $1000 \mathrm{M}_{\odot} \mathrm{yr}^{-1}$ ) should be condensing out of the ambient medium within an extended region of radius $100 \mathrm{kpc}$, activity at all other wavelengths is constrained to the central $10 \mathrm{kpc}$ (e.g., Hu et al. 1985; Heckman et al. 1989; McNamara \& O'Connell 1992; Donahue et al. 2000). With Chandra's subarcsecond resolution, even scales of $10 \mathrm{kpc}$ can be resolved in clusters at all redshifts.

${ }^{1}$ Harvard-Smithsonian Center for Astrophysics, 60 Garden St. Cambridge, MA 02138

${ }^{2}$ Department of Engineering Physics, University of Wollongong, Wollongong, NSW 2522, Australia

${ }^{3}$ Department of Physics and Astronomy, Ohio University, Athens, OH 45701

${ }^{4}$ School of Physics \& Astronomy, University of Birmingham, Birmingham B15 2TT, UK

${ }^{5}$ University of Washington, Astronomy Department, Box 351580, Seattle WA 98195

${ }^{6}$ Massachusetts Institute of Technology, Center for Space Research, 70 Vassar Street, Building 37, Cambridge, MA 02139
Some of the first results from Chandra show that the central regions of cooling flow clusters are not simple spherically symmetric systems, but are morphologically and spectroscopically very complex. Chandra has shown that radio emission from the central galaxy in cooling flows can have a significant impact on the X-ray morphology of the central regions. X-ray emission has been detected from the radio hotspots and the central point source in 3C295 (Harris et al. 2000; Wilson, Young \& Shopbell). Spectroscopic analysis of the Chandra data shows that the emission from the hotspots is probably due to synchrotron self-Compton and is not produced through an interaction between the relativistic plasma in the radio lobes and the thermal gas. The X-ray morphology of the central region of the Hydra A cluster (Abell 780) reveals the presence of cavities in the X-ray emitting gas coincident with the radio lobes (McNamara et al. 2000). The Chandra A observation of Hydra A also shows the presence of a heavily absorbed point source at the core of the radio galaxy (McNamara et al. 2000; Sambruna et al. 2000). The column density of absorbing material derived from spectral analysis of the central point source agrees with that observed in HI absorption (Taylor 1996). The Chandra observation of the core of the Perseus cluster shows a very complex X-ray morphology produced by interactions between the hot gas and the radio emitting plasma and also low energy absorption produced by an infalling gas rich galaxy (Fabian et al. 2000).

A massive cooling flow was reported in Hydra A based on Einstein (David et al. 1990), ROSAT (Peres et al. 1998), and ASCA (Ikebe et al. 1997) observations with estimated mass 
deposition rates ranging from 60 to $600 \mathrm{M}_{\odot} \mathrm{yr}^{-1}$. Einstein MPC and ASCA spectral analyses give an emission weighted temperature of $\sim 4 \mathrm{keV}$ (David et al. 1990; Peres et al. 1998). Temperature profiles have been derived from the ASCA data by Ikebe et al. (1997), Markevitch et al. (1998), and White (2000). The ASCA observation of Hydra A also reveals the presence of an Fe abundance gradient (Ikebe et al. 1997; Finoguenov, David, \& Ponman 2000) with a radially increasing silicon-toiron ratio indicating a predominance of Type II supernovae enriched material at large radii. From the Chandra observations we are able to spectroscopically determine the mass deposition rate, gas temperature, and abundance of heavy elements at a much greater spatial resolution than previously possible.

In the first paper on Hydra A, we concentrated on the interaction between the central FR Type I radio source (3C218) and the ambient cluster gas. In this paper we concentrate on the spectroscopic and morphological properties of the cooling flow region, derive high resolution temperature and abundance profiles of the gas within the central $250 \mathrm{kpc}$, and determine the total gravitating mass distribution in the core of the cluster. In addition, we compare the observed mass distribution in Hydra A with theoretical expectations from numerical simulations of dark matter halos (e.g., Navarro, Frenk, \& White 1997; Moore et al. 1998).

The remainder of this paper is organized as follows. In $\S 2$ we discuss the large scale X-ray morphology of the Hydra A cluster, the surface brightness profile, and the gas density distribution. In $\S 3$ we present the spectroscopic results concerning the temperature and abundance profiles of the hot gas. The total gravitating mass and gas mass distributions are derived in $\S 4$ along with a comparison with theoretical predictions. The roles of weak and strong shock heating by the central radio source in producing a convectively unstable region within the cooling flow are discussed in $\S 5$ and the main results are summarized in $\S 6$. We assume $\mathrm{H}_{0}=70 \mathrm{~km} \mathrm{~s}^{-1} \mathrm{Mpc}^{-1}, \Omega_{M}=0.3$, and $\Omega_{\Lambda}=0.7$ throughout the paper. At a redshift of 0.0538 , the luminosity distance to Hydra A is $240 \mathrm{Mpc}$, and $1^{\prime \prime}=1.05 \mathrm{kpc}$.

\section{X-RAY MORPHOLOGY AND SURFACE BRIGHTNESS}

Hydra A was observed during the orbital activation and calibration phase of the Chandra mission with both ACIS-I (October 30, 1999) and ACIS-S (November 2, 1999). Due to flares in the background charged particle flux, the data were screened to exclude all time intervals when the background rate was more than than $20 \%$ higher than the quiescent rate. The resulting screened integration times are 24,100 s and 18,950 s for the ACIS-I and ACIS-S observations. Only the standard grade set $(0,2,3,4,6)$ was used in our analysis. The gain was corrected using the CIAO task acis_process_events with the most recent version of the gain table appropriate for observations taken at a focal plane temperature of $-110^{\circ} \mathrm{C}$ (acisD1999-0916gainN0004.fits). This task also corrects for charge transfer inefficiency (CTI) effects in the front illuminated (FI) chips.

In McNamara et al. (2000), we showed a high resolution image of the combined ACIS-I and ACIS-S observations of Hydra A to illustrate the interaction between the radio lobes and the ambient cluster gas. To illustrate the large scale X-ray morphology of Hydra A we use only the ACIS-I observation due to the larger field of view and lower background. A background ACIS-I image was generated by co-adding observations taken at a focal plane temperature of $-110^{\circ} \mathrm{C}$ in the Chandra public archive after removing point sources from the observations. The same gain corrections and background screening criteria were applied to the observations resulting in a total integration time of $73 \mathrm{ksec}$ for the background image. The background image was then mapped onto the sky with the same aspect solution as in the Hydra A observation. The background subtracted image was corrected for detector and vignetting effects using an exposure map generated by convolving the aspect histogram with the mirror effective area and ACIS-I QE maps. Finally, the background-subtracted and exposure-corrected image was smoothed with a $\sigma=4^{\prime \prime}$ Gaussian. Contours of the smoothed ACIS-I image are shown in Figure 1 overlaid on the optical image of the cluster. The complex X-ray morphology within the central region discussed in McNamara et al. (2000) is evident in Figure 1. Beyond the central region, the X-ray morphology of Hydra A is fairly smooth with slightly elliptical contours, indicating that the cluster has not experienced a major merger in the recent past. The X-ray isophotes are aligned in the same SE-NW direction and have the same ellipticity as the I-band isophotes shown in McNamara (1995). There is thus a remarkably strong alignment between the stellar and gaseous components in the Hydra A cluster from scales of a few kpc up to $300 \mathrm{kpc}$.

While the ACIS-I observation is useful for illustrating the large scale 2-D morphology of Hydra A, we use the ACIS-S observation to derive an X-ray surface brightness profile due to the better photon statistics on the back-illuminated (BI) S3 chip. The BI chips on ACIS have higher QEs than the FI chips for photon energies below $4 \mathrm{keV}$. A broad band $(0.3-7.0 \mathrm{keV})$ surface brightness profile derived from the $\mathrm{S} 3$ data is shown in Figure 2. A similar procedure was used to perform the background subtraction as discussed above for the the ACIS-I data by co-adding $106 \mathrm{ksec}$ of ACIS-S3 data taken at a focal plane temperature of $-110^{\circ} \mathrm{C}$. The same reductions were applied to this background image as discussed above for the ACIS-I background image. Background count rates were then obtained from the same annuli as the total count rates. The origin in the surface brightness profile corresponds to the location of the central point source. The annuli were chosen to ensure approximately 2,000 net counts per annulus. Chandra's high resolution shows that there are two inflection points in the surface brightness profile at approximately $100 \mathrm{kpc}$ and $200 \mathrm{kpc}$. It is obvious from Figure 2 that neither a single nor a double $\beta$ model will provide a good fit to the data. The break in the surface brightness profile at $100 \mathrm{kpc}$ is also evident in the ROSAT PSPC observation discussed by Mohr, Mathiesen \& Evrard (1999). Beyond the central $200 \mathrm{kpc}$, the surface brightness is approximately characterized by a power-law with a slope corresponding to $\beta=0.7$, in good agreement with the Einstein IPC results (David et al. 1990) and the ROSAT PSPC results (Mohr et al. 1999; Vikhlinin, Forman, \& Jones 1999).

Due to the complexity of the surface brightness in the central region of Hydra A, we use a deprojection technique to determine the gas density profile rather than fitting a multivariate function. The deprojection technique first determines the density of the gas in the largest annulus using the observed count rate and an assumed emissivity and then determines the density at progressively smaller radii after removing the projected emission from gas at larger radii. The fraction of the cluster emission in the outermost annulus arising from gas at larger radii is corrected using the best fit $\beta$ model to the PSPC observation of Hydra A in Mohr, Mathiesen \& Evrard (1999). We perform the deprojection 100 times using a Monte Carlo technique, that randomizes the surface brightness profile based on the observed errors. The emissivity is assumed to be that of 
a $3.5 \mathrm{keV}$ gas with a $40 \%$ solar abundance of heavy elements. In $\S 4$ below we show that the gas temperature varies between 3.0 and $4.0 \mathrm{keV}$ in this region and that the abundance varies between 0.2 and 0.6 solar. The assumption of a spatially independent gas emissivity only introduces a $3 \%$ uncertainty in the gas density. The mean gas densities and rms deviations from the the 100 Monte Carlo simulations are shown in Figure 3. This figure shows that the density profile gradually steepens from $\rho_{\text {gas }} \propto r^{-0.4}$ at $30 \mathrm{kpc}$ to $\rho_{\text {gas }} \propto r^{-1.6}$ at $100 \mathrm{kpc}$, and there is no obvious flat density core.

The isobaric cooling time, $t_{c}$, of the gas is shown in Figure 4. Note that $t_{c}$ is less than a Hubble time $\left(\sim 10^{10} \mathrm{yr}\right)$ within the central $200 \mathrm{kpc}$, and less than $1 \mathrm{Gyr}$ within the central $30 \mathrm{kpc}$. This inner radius is important for the discussion below and appears to be the outer boundary of an isentropic core of multiphase gas.

\section{SPECTRAL ANALYSIS}

The ACIS-S observation of Hydra A produced 160,000 source counts within the central $250 \mathrm{kpc}$ on the $\mathrm{S} 3$ chip. Spectra were extracted in 8 concentric annuli centered on the point source with widths varying from $10^{\prime \prime}$ to $30^{\prime \prime}$ to ensure 20,000 source counts per spectrum. Spectra also were extracted with 40,000 net counts each, since better photon statistics are required to determine the Si distribution. We excised the emission from the central point source using a $2^{\prime \prime}$ radius circle. Background spectra were extracted from the same regions in the background fits event list and were normalized by exposure time. All spectra were extracted in PI space and rebinned to a minimum of 20 counts per bin. A single PI rmf file was generated using mkrmf and the FEF file appropriate for the aim point on S3, node 1, at a focal plane temperature of $-110^{\circ} \mathrm{C}$. Area files were generated for each spectrum based on the mean off-axis angle of the extracted photons in each spectrum. We fitted each spectrum to a variety of XSPEC models: an absorbed single temperature model, an absorbed single temperature plus cooling flow model, an absorbed single temperature plus intrinsically absorbed cooling flow model, and an absorbed two temperature model (see Tables 1-3). Only data between 0.5$8.0 \mathrm{keV}$ were included in the analysis. Below $0.5 \mathrm{keV}$ the detector gain is nonlinear which is not yet properly treated in the response matrices. Above $8.0 \mathrm{keV}$, the charged particle background dominates the source emission.

The ACIS-I data were extracted in a similar fashion, however, several modifications are required to account for the nonuniformity of the FI chips due to radiation induced CTI effects. Instead of a single rmf, a photon-weighted rmf was generated for each spectrum. In addition, the CTI damage to the ACIS-I chips produces a change in quantum efficiency at high energies (events far from the chip readout experienced significant grade migration, transforming acceptable grades to unacceptable grades) that we corrected using the ACIS-I QE maps at $\mathrm{T}=-110^{\circ} \mathrm{C}$.

The spectral fitting results for the inner three annuli of the ACIS-S data are shown in Tables 1-3 and Figures 5-7. The spectrum from the inner $2^{\prime \prime}-17^{\prime \prime}$ is the most complex, and significant reduction in $\chi^{2}$ can be obtained by adding multiple spectral components. Table 1 shows that including a cooling flow component in the inner spectrum improves the fit with a reduction in $\chi^{2}$ of 5 to 8 depending on whether $N_{H}$ is fixed to the galactic value or allowed to vary. However, there is not an obvious improvement when simply examining the best-fit of the two models to the data (see Figure 5). The spectral analysis in the innermost annulus where the cooling time is $\sim 10^{8} \mathrm{yrs}$, shows that less than $24 \%$ (at the $90 \%$ confidence limit) of the total emission arises from gas condensing out of the flow. While the absorbed cooling flow model produces the minimum $\chi^{2}$ and largest $\dot{\mathrm{M}}$, the best-fit galactic $N_{H}$ in this model is more than a factor of two below the Stark et al. (1992) value. The intrinsic and galactic absorption are strongly correlated in the fitting process, and it is difficult to distinguish between them when fitting the spectrum of a low-redshift, low-M cluster. Fixing $N_{H}$ to the galactic value significantly reduces $\dot{M}$ and limits the intrinsic absorption to $<3.2 \times 10^{20} \mathrm{~cm}^{-2}$ (see Table 1). Adding a cooling flow component to the second annulus only provides a slight improvement to the fit (see Table 2), while adding a cooling flow component to the third annulus does not produce any improvement (see Table 3). Figures 6 and 7 show how well the spectra from the second and third annuli are fit with a single temperature model. Beyond the central $30 \mathrm{kpc}$, all of the spectra are well fit with single temperature models and the addition of more free parameters does not significantly improve the fits.

The lack of a strong spectroscopic signature for a massive cooling flow is not due to the small spatial binning of the spectra. In Figure 8 we show a spectrum extracted from the central $100 \mathrm{kpc}$, excluding the central point source, fit to a single temperature plus cooling flow model with $\dot{M}$ fixed at $140 \mathrm{M}_{\odot} \mathrm{yr}^{-1}$ (the value obtained from a deprojection of the ROSAT PSPC data by Peres et al. [1998] after adjusting to our adopted cosmology). All other parameters are treated as free parameters. Figure 8 shows that there are very large residuals below $2 \mathrm{keV}$ and the resulting $\chi^{2} / \mathrm{DOF}=1175 / 355$. Our results are in reasonable agreement with the spectral analysis of the ASCA SIS data on Hydra A by Ikebe et al. (1997) who obtained $\dot{\mathrm{M}}=30 \pm 15 \mathrm{M}_{\odot} \mathrm{yr}^{-1}$ (scaled to our adopted cosmology). The implications of these results for cooling flows will be discussed below.

\subsection{Temperature Profile}

The gas temperature profile derived from the ACIS-S and ACIS-I data is shown in Figure 9. The inner two temperature points for the ACIS-S data were derived from the best-fit to a single absorbed temperature plus cooling flow model. The remaining temperature points for the ACIS-S data were obtained without a cooling flow component in the spectral analyses. All ACIS-I spectra were fitted to single temperature models with $N_{H}$ fixed to the galactic value and a variable abundance of heavy elements. Figure 9 shows that there is good agreement between the two ACIS detectors and that the gas temperature in Hydra A increases from 3 to $4 \mathrm{keV}$ within the central $200 \mathrm{kpc}$ as noted in McNamara et al. (2000). It is very difficult to obtain any temperature estimates from the ACIS-S data beyond $200 \mathrm{kpc}$ due to the high background in the S3 chip; however, the ACIS-I data show that the temperature drops to about $3 \mathrm{keV}$ at $400-600 \mathrm{kpc}$ . Previous temperature profiles for Hydra A were derived from ASCA data by Ikebe et al. (1997), Markevitch et al. (1998), and White (2000). The primary difference between the Chandra and ASCA profiles is the lower gas temperature in the center of the cluster and the steeper temperature gradient. This is to be expected given the greater spatial resolution of Chandra.

\subsection{Abundance Gradients}

Intermediate temperature clusters like Hydra A emit strongly in both $\mathrm{Fe} \mathrm{L}$ and $\mathrm{Fe} \mathrm{K}$ lines. To determine the $\mathrm{Fe}$ abundance of the gas, we fitted the ACIS-S spectra in a broad energy band 
from $0.5-7.5 \mathrm{keV}$, that includes emission from both $\mathrm{Fe} \mathrm{L}$ and $\mathrm{K}$ lines, and a hard band from 2.0-7.5 keV, that only includes Fe K lines. The best-fit $\mathrm{Fe}$ abundances are shown in Figure 10 based on an absorbed VMEKAL model with $N_{H}$, all $\alpha$-processed elements, and Fe treated as free parameters. This figure shows that there is excellent agreement between these two determinations and that there is a strong $\mathrm{Fe}$ abundance gradient within the central $100 \mathrm{kpc}$. The agreement between the two Fe estimates shows that potential low energy complications, e.g., excess absorption or cooling gas, do not have a significant impact on the Fe abundance measurements. An Fe abundance gradient in Hydra A also was reported based on ASCA data by Ikebe et al. (1997) and Finoguenov, David, \& Ponman (2000). While the magnitude of the measured $\mathrm{Fe}$ gradient is comparable between the ASCA and Chandra analysis, only Chandra has the spatial resolution to resolve the Fe distribution within the central few arcminutes. Figure 10 shows that the $\mathrm{Fe}$ gradient is confined to the central $100 \mathrm{kpc}$, while analysis of the ASCA data implied a flatter gradient extending to $200-300 \mathrm{kpc}$.

Significant constraints on the Si abundance can only be obtained from the set of spectra with 40,000 net counts. Figure 11 shows that the Si distribution is similar to the Fe distribution with a rapid decline within the central $100 \mathrm{kpc}$. Beyond $100 \mathrm{kpc}$, the Si abundance is essentially constant within the uncertainties. Due to the large errors on the $\mathrm{Si}$ abundance, it is difficult to make conclusive comments on the radial variation in the $\mathrm{Si} / \mathrm{Fe}$ abundance ratio.

\subsection{Deprojected Temperature and Entropy Profiles}

The projected temperature profile shows that there is a strong positive gradient within the central $200 \mathrm{kpc}$ (see Figure 9). To determine the deprojected temperature profile in this region, we first fitted the outer ACIS-S spectrum to a single MEKAL model, with $N_{H}$ and the abundance of heavy elements treated as free parameters. We then fitted the spectrum extracted from the next smallest annulus to a two temperature model, with the parameters of one of the models frozen to the best-fit values derived from the outermost spectrum. The normalization of this model is adjusted to account for the volume within the outer shell projected along the line of sight toward the next smallest shell. This procedure is continued inward, adding a MEKAL model for each successive spectrum, with the free parameters of the remaining models frozen to the previously determined best-fit values. Finally, the best-fit temperatures, along with their associated errors, are fit to a power-law profile given by $T(r)=T_{0}(r / 10 \mathrm{kpc})^{\mathrm{p}}$. The best-fit is obtained with $T_{0}=2.73 \pm 0.07 \mathrm{keV}$ and $p=0.124 \pm 0.014$. The gas entropy is determined from the deprojected density profile and the bestfit power-law temperature profile (see Figure 12). This figure shows that the gas is approximately isentropic within the central $30 \mathrm{kpc}$, and the gas entropy only increases significantly at larger radii.

\section{MASS DISTRIBUTIONS}

The gravitating mass distribution is determined from the equation of hydrostatic equilibrium, given by:

$$
M_{t o t}(<r)=-\frac{k T r}{\mu m_{p} G}\left(\frac{d \ln \rho_{g a s}}{d \ln r}+\frac{d \ln T}{d \ln r}\right)
$$

We use a parametric form for the gas temperature (the best power-law profile given above) along with the nonparametric density profile shown in Figure 3 to determine the mass distri- bution in Hydra A. To reduce the noise in the $\mathrm{d} \ln \rho_{\text {gas }} / \mathrm{d} \ln \mathrm{r}$ term, we determine the gradient at $r_{j}$ by differencing the densities at $r_{j-2}$ and $r_{j+2}$. The density gradients are assumed to be equal in the innermost 3 points and the outermost 3 points. The cumulative gravitating mass, gas mass, $\mathrm{Fe}$ mass, and $\mathrm{Si}$ mass are shown in Figure 13. In addition, we show the gravitational potential in Figure 14, with the potential normalized to zero at $250 \mathrm{kpc}$. Of the four mass components shown in Figure 13, the $\mathrm{Fe}$ and $\mathrm{Si}$ are the most centrally concentrated, the gas is the most extended, and the dark matter is intermediate between the two. The central concentration of a given mass component can be quantized by calculating the ratio of the mass within 100 $\mathrm{kpc}$ to that within $200 \mathrm{kpc}$. For the gas and dark matter this ratio is 27\% and 33\%, respectively. Also shown in Figure 13 are the integrated $\mathrm{Fe}$ and $\mathrm{Si}$ masses that would be derived under the assumption of a uniform distribution of metals using the best-fit values derived from a spectral analysis of the integrated emission within the central $200 \mathrm{kpc}$. This figure shows that the assumption of a uniform distribution of heavy elements underestimates the $\mathrm{Fe}$ and $\mathrm{Si}$ masses by a factor of two at small radii and overestimates the $\mathrm{Fe}$ and $\mathrm{Si}$ masses by approximately $30-50 \%$ at large radii. This has important consequences concerning the chemical enrichment history of clusters.

The variation in the gas mass fraction with radius is shown in Figure 15. This figure shows that the gas mass fraction increases from $4 \%$ at $10 \mathrm{kpc}$ (several times greater than a typical isolated giant elliptical) up to $15 \%$ at $200 \mathrm{kpc}$, which is typical of rich clusters (David, Jones, \& Forman 1995; White $\&$ Fabian 1995; Evrard 1997). The effects of the cluster environment on the X-ray characteristics of the central dominant galaxy will be discussed in $\S 6$. The blue light within the central $47 \mathrm{kpc}$ of Hydra A is $L_{B}=9.2 \times 10^{10} L_{\odot}($ see $\S 6)$ which gives $M / L_{B}=76 \mathrm{M}_{\odot} / \mathrm{L}_{\odot}$. Assuming $M / L_{B}=8 \mathrm{M}_{\odot} / \mathrm{L}_{\odot}$ for the stellar population of Hydra A gives a stellar mass fraction of $10 \%$ for a total baryon fraction of $15 \%$. The total baryon fraction within the central $47 \mathrm{kpc}$ is thus comparable to the gas mass fraction beyond $100 \mathrm{kpc}$.

\subsection{Comparison with Theoretical Expectations}

There has been a great deal of discussion in the literature concerning the expected density profile of CDM halos and the observed density distribution of galaxies and clusters. Based on an extensive set of numerical simulations of the formation and evolution of dark matter halos, Navarro, Frenk \& White $(1995,1998)$ concluded that dark matter halos have a universal density profile given by:

$$
\frac{\rho_{d}}{\rho_{\text {crit }}(z)}=\frac{\delta_{c}}{\left(\frac{r}{r_{s}}\right)^{\gamma}\left(1+\left(\frac{r}{r_{s}}\right)^{\alpha}\right)^{(\nu-\gamma) / \alpha}}
$$

where $\alpha=1, \nu=3, \gamma=1, \rho_{\text {crit }}(z)=3 H^{2} / 8 \pi G$ is the critical density of the universe at the cluster redshift, $r_{s}$ is a characteristic radius, and $\delta_{c}$ is the central overdensity which can be expressed in terms of the concentration parameter $\left(c=r_{200} / r_{s}\right.$; where $r_{200}$ is the radius within which the mean halo density is $\left.200 \rho_{\text {crit }}\right)$ as

$$
\delta_{c}=\frac{200}{3} \frac{c^{3}}{[\ln (1+\mathrm{c})-\mathrm{c} /(1+\mathrm{c})]} .
$$

The NFW density profile varies from $\rho_{d} \propto r^{-1}$ at small radii to $\rho_{d} \propto r^{-3}$ at large radii. For a given cosmology, the concentration parameter decreases with increasing halo mass. Higher 
resolution simulations by Moore et al. (1998) found a density profile similar to the NFW profile at large radii, but with a steeper density profile at small radii $\left(\rho_{d} \propto r^{-1.5}\right)$ corresponding to $\alpha=1.5, \nu=3$, and $\gamma=1.5$. Jing (2000) examined how the density profile depends on the dynamic state of dark matter halos. He found significant variations in the density profile of halos at the present epoch in his simulations. By fitting the simulated halo density profiles with an NFW profile, he found that the observed distribution in the concentration parameter can be described by a lognormal function. Virialized systems have small residuals when fit with the NFW profile and a mean concentration parameter comparable to that predicted by NFW. Unrelaxed systems produce larger residuals and smaller concentration parameters when fit to the NFW profile.

The primary difficulty encountered when these theoretical predictions for CDM halos are compared to observations is the inferred presence of flat cores in the density profiles of dwarf and low surface brightness galaxies (Flores \& Primack 1994; Burkert 1995; Moore et al. 1998). For example, Moore et al. showed that the HI rotation curves of low surface brightness galaxies are best fit with a modified King profile $(\alpha=2, \nu=3$, and $\gamma=0$ ). However, van den Bosch et al. (2000) argue that the resolution of the present $\mathrm{HI}$ data are insufficient to decouple the disk and halo components of low surface brightness galaxies and cannot be used to place significant constraints on the central density distribution. They suggest that flat cores may only exist in low mass dwarf systems, that are more susceptible to the effects of supernovae driven winds. A recent paper by Swaters, Madore, \& Trewhella (2000) demonstrates that high resolution $\mathrm{H}_{\alpha}$ rotation curves of low surface brightness galaxies do indeed increase faster with radius than lower resolution HI rotation curves. On cluster scales, Tyson, Kochanski, \& Dell'Antonio (1998) analyzed strong lensing data on CL $0024+1654$ and found evidence for a flat core, while Broadhurst et al. (2000) concluded that a flat core is not required by the strong lensing data for this cluster. However, Shapiro, \& Iliev (2000) show that the mass distribution used by Broadhurst et al . predicts a galaxy velocity dispersion much greater than that observed, while the mass distribution derived by Tyson et al. is consistent with the observed velocity dispersion.

The cumulative gravitating mass distribution of the Hydra A cluster in Figure 13 shows that there is little evidence for a flat core with a core radius greater than approximately $30 \mathrm{kpc}$. We cannot exclude the presence of a smaller core, since we are only able to constrain the gas temperature within two annuli in this region (see Figure 9). Between 30 and $200 \mathrm{kpc}$, the integrated mass scales as $M(<r) \propto r^{1.7}$, or equivalently, $\rho_{d} \propto r^{-1.3}$, which is slightly steeper than the NFW profile and slightly flatter than the Moore profile. The mass profile is actually in very good agreement with simulations that incorporate the effects of gas cooling and star formation by Lewis et al. (2000), who find $\rho_{d} \propto r^{-1.4}$. Fitting the mass distribution in Hydra A to these analytic models we obtain best-fit parameters of $\delta_{c}=(7.48 \pm 0.14) \times 10^{4}$ and $r_{s}=77 \pm 10 \mathrm{kpc}, \delta_{c}=$ $(4.02 \pm 0.36) \times 10^{3}$ and $r_{s}=234 \pm 25 \mathrm{kpc}$, and $\delta_{c}=4.54 \times 10^{5}$ and $r_{s}=26 \pm 2 \mathrm{kpc}$, for the NFW, Moore, and modified King profiles, respectively. The best-fit $\delta_{c}$ uniformly decreases with the level of mass concentration in the models. Eq. (3) is strictly valid only for the NFW profile and yields a concentration parameter of $c_{N F W}=12.3 \pm 0.18$. The best fit profiles for the three mass models are shown along with the cumulative gravitating mass in Figure 16. This figure shows that both the NFW and Moore profiles provide reasonable fits to the data, supporting the results of numerical CDM simulations. Based on the rotation curve of the $H_{\alpha}$ disk in Hydra A published in Melnick et al. (1997), the gravitating mass within $3.7 \mathrm{kpc}$ is $3.9-7.8 \times 10^{10} \mathrm{M}_{\odot}$, for inclination angles ranging from 0 to $45^{\circ}$. The addition of this point in Figure 16 favors the NFW profile. The gravitational potential of the central galaxy should not affect our results since the gas temperature at $10 \mathrm{kpc}$ is $2.7 \mathrm{keV}$. This is significantly hotter than the virial temperature of the central galaxy ( $k T \approx 0.6 \mathrm{keV}$ ) derived from the stellar velocity dispersion of $307 \mathrm{~km} \mathrm{~s}^{-1}$ (McElroy 1995).

The Hydra A cluster is approximately a $10 M_{*}$ cluster. For the CDM $\Lambda$ cosmology used by NFW (essentially the same as that used to estimate distance dependent quantities in this paper) the predicted concentration parameter for a $10 M_{*}$ halo is 4.0 , which is only one-third of the observed value. The high observed concentration of the Hydra A cluster implies an improbable collapse redshift of $z \approx 4$ (using the expression given in the appendix of NFW). The high concentration parameter in Hydra A cannot be resolved with NFW by appealing to the possible presence of residual substructure since Jing (2000) finds that unrelaxed clusters yield concentration parameters lower than the NFW values. Based on the NFW simulations, a concentration parameter of $c_{N F W} \approx 12$ is expected for a $0.01 M_{*}$ dark matter halo, which actually corresponds to the integrated mass within the central $10 \mathrm{kpc}$ of Hydra A. In summary, we find that the total density distribution in the central region of the Hydra A cluster is fairly steep with $\rho_{d} \propto r^{-1.3}$. The observed mass distribution is in reasonable agreement with CDM simulations, but the derived concentration parameter is more representative of a galaxy-size halo rather than a rich cluster of galaxies. This analysis will be extended to a sample of rich clusters in Robertson \& David (2000).

\section{EVIDENCE FOR FEEDBACK IN THE COOLING FLOW}

The morphological mass accretion rate across a spherical shell $\mathrm{j}$ can be estimated from:

$$
\dot{M}_{j}=\frac{L_{j}}{\left(\Delta H_{j}+\Delta \phi_{j}\right)}
$$

where $L_{j}$ is the deprojected X-ray luminosity within shell $\mathrm{j}$, and $\Delta H_{j}$ and $\Delta \phi_{j}$ are the changes in gas enthalpy and gravitational potential across shell j. Eq. (4) gives the mass accretion rate assuming steady-state and no mass deposition, in agreement with the spectroscopic results beyond $30 \mathrm{kpc}$. This equation differs from that used in, e.g., Allen (2000) because we do not include the X-ray emission arising from gas condensing out of the flow. Figure 17 shows the resulting morphological M profile using the deprojected X-ray luminosity, the best-fit power-law temperature profile, and the gravitational potential shown in Figure 14. Eq. (4) is only strictly valid beyond $30 \mathrm{kpc}$ and the only self-consistent solution is one with a constant $\dot{M}$, which is in fact the derived solution. Beyond $30 \mathrm{kpc}$, the morphological $\dot{\mathrm{M}}$ is nearly constant at $300 \mathrm{M}_{\odot} \mathrm{yr}^{-1}$ (see Figure 17). This result provides strong evidence that there is a steady, (nearly) homogeneous cooling flow beyond $30 \mathrm{kpc}$. Inside $30 \mathrm{kpc}$, there is spectroscopic evidence for multiphase gas, but the spectroscopic mass deposition rate is more than a factor of 10 less than the morphological mass accretion rate at $30 \mathrm{kpc}$. Figure 17 also shows that the $\dot{M}$ calculation is not very sensitive to the details of the gravitational potential. The change in gas enthalpy within the central $200 \mathrm{kpc}$ is $2.5 \mathrm{keV}$ per particle, while the change in gravitational potential is only $0.5 \mathrm{keV}$ per particle (see Figure 
14).

The discrepancy between the morphological mass accretion rate at $30 \mathrm{kpc}$ and the spectroscopic $\dot{M}$ within this radius suggests that some heating mechanism may be suppressing the accretion of the cooling gas. Many heating mechanisms have been proposed over the past two decades in the hopes of significantly reducing the morphological mass accretion rate of cooling flow clusters. One of the most promising is heating from a central radio source, since approximately $70 \%$ of cooling flow clusters have active central galaxies. Heinz, Reynolds, \& Begelman (1998) showed that shock heating by expanding relativistic plasma can induce observable X-ray features in the ambient cluster gas. Tucker \& David (1997) showed that collective heating effects from relativistic plasma can generate a duty cycle between accretion and outflow that can significantly reduce the integrated mass accreted over the lifetime of a cluster. A similar model based on Compton heating by a central AGN in ellipticals has been developed by Ciotti \& Ostriker (1999). Recently, Soker et al. (2000) proposed a moderate cooling flow model due to heating by radio sources that primarily affects the cooling rate of gas in the outer regions of cooling flows, significantly reducing their time-averaged mass accretion rates. In addition to direct heating, Churazov et al. (2000) has shown that hot buoyant bubbles produced through shock heating by the expanding central radio source can dredge up cold material from the center of the cluster. In this paper we further examine the role of weak and strong shock heating by a central radio source and discuss the development of a convectively unstable region in cooling flows that can significantly reduce the net inflow of cooling gas.

\subsection{Heating by Weak Shocks}

The lack of evidence for gas deposition and the flattening of the entropy profile near the center of the cluster suggest that the cluster gas is being heated appreciably by the radio source. On the other hand, if this is the case, then the temperature decline towards the center of the cluster is surprising, since it is an indication of cooling. If the age of this cluster since its last major merger is about $10^{10} \mathrm{yr}$, then, in order to prevent the deposition of $300 \mathrm{M}_{\odot} \mathrm{yr}^{-1}$ of cooled gas, the heating power needs to make up for the radiative heat loss within the central $150 \mathrm{kpc}$ of the cluster, i.e., about $2.5 \times 10^{44} \mathrm{erg} \mathrm{s}^{-1}$. This is plausible, since it is only 4 times the current radio power (Ekers \& Simkin 1983). Here we consider some of the details of how heating by shocks from the radio source can prevent gas deposition.

It is generally argued that the mechanical power of expanding radio jets exceeds the radio power by a substantial factor. This leads to models in which the expanding radio jets drive a roughly spherical shock into the surrounding gas (Heinz, Reynolds, \& Begelman 1998). Shock heating due to such radio outbursts is a good candidate for the required heating. The typical lifetime of cluster center radio sources is $10^{7}-10^{8} \mathrm{yr}$ (e.g., Taylor et al. 1990), so that the high incidence of central radio sources in cooling flow clusters (Burns et al. 1997) requires that they undergo frequent repeated outbursts. We begin by considering the accumulated heating effect of repeated weak shocks due to these outbursts.

The shock jump conditions may be written as

$$
\frac{p_{1}}{p_{0}}=1+\frac{2 \gamma}{\gamma+1} y
$$

and

$$
\frac{\rho_{1}}{\rho_{0}}=\frac{(\gamma+1)(1+y)}{\gamma+1+(\gamma-1) y}
$$

where $p$ is the pressure, $\rho$ the density, $\gamma$ the ratio of specific heats, and subscripts ' 0 ' and ' 1 ' refer to pre-shock and postshock conditions, respectively. The quantity $y=\rho_{0} v_{\mathrm{s}}^{2} /\left(\gamma p_{0}\right)-$ 1 is the square of the shock Mach number minus 1 , so that $y \ll 1$ for weak shocks and $y \gg 1$ for strong shocks. The specific entropy jump for a weak shock is then

$$
\Delta S \simeq \frac{2 \gamma k}{3(\gamma+1)^{2} \mu m_{\mathrm{H}}} y^{3} .
$$

For a weak shock the kinetic energy of the shocked gas is negligible compared to its increase in thermal energy. Assuming that the postshock pressure excess is roughly uniform, we can therefore estimate the shock strength by equating the mechanical energy that drives the shock to the excess thermal energy within the volume $V$ encompassed by the shock, i.e.,

$$
E_{\mathrm{T}} \simeq \frac{1}{\gamma-1}\left(p_{1}-p_{0}\right) V
$$

Using the shock jump condition for $p_{1}$, this gives an expression for $y$, which determines the specific entropy jump in the shock

$$
\Delta S \simeq \frac{(\gamma-1)^{3}(\gamma+1) k}{12 \gamma^{2} \mu m_{\mathrm{H}}}\left(\frac{E_{\mathrm{T}}}{p_{0} V}\right)^{3} .
$$

The requirement that the shock is weak is $E_{\mathrm{T}} \ll p_{0} V$. If the average repetition frequency of outbursts is $1 / \tau$, then the average heating rate per unit volume due to weak shocks is

$$
H=\rho_{0} T_{0} \frac{\Delta S}{\tau}=\frac{(\gamma-1)^{3}(\gamma+1) p_{0}}{12 \gamma^{2} \tau}\left(\frac{E_{\mathrm{T}}}{p_{0} V}\right)^{3} .
$$

Comparing this to the radiative cooling rate per unit volume, $R=\rho_{0}^{2} \Lambda\left(T_{0}\right)$, gives

$$
\frac{H}{R}=\frac{(\gamma-1)^{4}(\gamma+1)}{12 \gamma^{2}}\left(\frac{t_{\mathrm{c}, 0}}{\tau}\right)\left(\frac{E_{\mathrm{T}}}{p_{0} V}\right)^{3},
$$

where the cooling time is $t_{\mathrm{c}, 0}=p_{0} /[(\gamma-1) R]$.

Taking $\Lambda(T) \propto T^{\alpha}$, the ratio $H / R \propto \rho_{0}^{-4} T_{0}^{-(2+\alpha)} r^{-9}$, so that, if the gas is nearly isothermal, $H / R$ increases with $r$ only if $\rho_{0}$ decreases more rapidly than $r^{-9 / 4}$. This is significantly steeper than the observed $r$ dependence of $\rho$ in the region of interest, so that $H / R$ is a decreasing function of $r$ within the central 150 kpc of the Hydra A cluster. Thus, heating by weak shocks can only dominate cooling at small $r$. The shock heating rate would dominate the cooling rate everywhere inside the point where they balance, i.e., $r=r_{\mathrm{b}}$, where $H=R$. At small $r$ the net heating time, $p_{0} /[(\gamma-1)(H-R)] \simeq p_{0} /[(\gamma-1) H]$, would then be shorter than the cooling time and the gas is heated rapidly. Where the net heating is substantial it leads to convective instability because the heating time is shortest at small $r$. Where the heating is not too fast (which includes the region where the shocks are weak), convective instability drives mixing that will result in a nearly isentropic core.

Details of the process leading to convection are complex, since as the gas is heated it is redistributed and the pressure and density profiles are flattened in order to maintain hydrostatic equilibrium. At $r_{\mathrm{b}}$ the entropy of the gas remains fixed. To get a rough idea of what part of the gas becomes isentropic we use the crude estimate 


$$
t_{\mathrm{S}}=\frac{S\left(r_{\mathrm{b}}\right)-S(r)}{d S /\left.d t\right|_{r}}
$$

for the time it takes for the entropy of the gas at $r$ to rise to the entropy of gas at $r_{\mathrm{b}}$. Assuming that the gas is (initially) isothermal and $\rho_{0}(r) \propto r^{-\beta}$, the results above then give

$$
t_{\mathrm{S}}=\frac{(\gamma-1) \beta \ln \left(r_{\mathrm{b}} / r\right)}{\left(r_{b} / r\right)^{9-4 \beta}-1} t_{\mathrm{c}, 0}(r) .
$$

Taking $\beta=1.5$, roughly appropriate for the region outside the isentropic core in Hydra, this gives $t_{\mathrm{S}} \simeq 0.1 t_{\mathrm{c}, 0}$ at $r=r_{\mathrm{b}} / 2$. Since the cooling time is already relatively short in this region, this suggests that the bulk of the gas within $r_{\mathrm{b}}$ should be isentropic.

\subsection{Heating by Strong Shocks}

For the purpose of this discussion, heating by strong shocks adds one significant complication, that a single strong shock can cause a substantial entropy rise. This can produce 'bubbles' of gas with such high entropy that they rise well outside the isentropic core region $\left(r_{\mathrm{b}}\right)$ before arriving at their equilibrium position. Convection still creates an isentropic core within a few free-fall times after passage of the shock, but some gas may rise well outside this core.

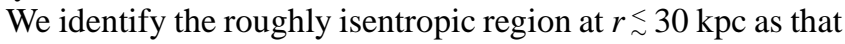
where the average shock heating rate exceeds radiative cooling. This determines the strength of shocks due to the radio outbursts as follows. The shock repetition time, $\tau$, is highly uncertain, but, as mentioned above, it needs to be comparable to the radio source lifetime to account for the high incidence of central radio sources in cooling flow clusters. Taking $\tau=10^{8} \mathrm{yr}$, the cooling time at $r=30 \mathrm{kpc}$ is too short relative to the shock repetition time for weak shocks to balance the radiative cooling rate. Instead we must appeal to stronger shocks to provide the heating, but in that case balancing the heating rate against the average cooling rate is complicated by the large excursions in gas properties caused by the shocks. Provided that the gas is reasonably close to hydrostatic equilibrium most of the time, we can use the ratio of the average rate of change of entropy due to shocks to that due to radiative heat loss, i.e.,

$$
\begin{gathered}
Q=\frac{\Delta S / \tau}{R /(\rho T)} \\
=\frac{t_{\mathrm{c}}}{\tau}\left[\ln \left(1+\frac{2 \gamma y}{\gamma+1}\right)+\gamma \ln \left\{\frac{\gamma+1+(\gamma-1) y}{(\gamma+1)(1+y)}\right\}\right] .
\end{gathered}
$$

This is essentially the same as the expression for $H / R$ above, except that here $t_{\mathrm{c}}$ is an 'average' cooling time, which we assume to be close to the observed cooling time, and the expression for the entropy jump in terms of $y$ is exact. Taking $t_{\mathrm{c}} / \tau \simeq 10$ at $r=30 \mathrm{kpc}$ and $\gamma=5 / 3$ then requires $y \simeq 2$ to make $Q=1$ there. If the observed pressure is close to the preshock pressure, $p_{0}$, equation (7) still gives a reasonable estimate for the shock energy. Taking $y=2, k T=3.3 \mathrm{keV}$ and $n_{\mathrm{e}}=0.027 \mathrm{~cm}^{-3}$, as observed at $r=30 \mathrm{kpc}$, we get $E_{\mathrm{T}} \simeq$ $3.4 \times 10^{60} \mathrm{erg}$. For $\tau=10^{8} \mathrm{yr}$, this makes the average shock power about $1.1 \times 10^{45} \mathrm{erg} \mathrm{s}^{-1}$. This is to be compared with the total power radiated by the gas within $r=150 \mathrm{kpc}$, i.e., about $2.5 \times 10^{44} \mathrm{erg} \mathrm{s}^{-1}$. Given the large uncertainty in the shock energy, the agreement is close, suggesting that the radiative cooling rate in the central $150 \mathrm{kpc}$ and shock heating due to radio outbursts are tied together. Note that these numbers agree for $\tau \simeq 4 \times 10^{8} \mathrm{yr}$.

Direct shock heating does not fully explain the lack of gas deposition, since the argument above suggests that it is insignificant compared to radiative cooling outside the central $30 \mathrm{kpc}$. In that case, with no heat source to make up for radiative losses, cooling gas from the region between 30 and $150 \mathrm{kpc}$ flows into the central $30 \mathrm{kpc}$ at about $300 \mathrm{M}_{\odot} \mathrm{y}^{-1}$. If the system is to remain in an approximately steady state, then an equivalent amount of gas must be driven back into the region surrounding the core by each radio outburst. Again, taking $\tau \simeq 10^{8} \mathrm{yr}$, this means that each radio outburst must drive about $3 \times 10^{10} \mathrm{M}_{\odot}$ of gas from the core. This is roughly $30 \%$ of the mass of gas in the $30 \mathrm{kpc}$ core. The core gas is roughly isentropic, so a strong shock can certainly cause a substantial entropy inversion, i.e., gas that can rise well outside the core. It is not clear that as much as 30\% of the gas can be lifted from the core by a single shock. More accurate numbers and more detailed modeling are needed to determine if this is possible.

The detectability of the hot bubbles rising from the core is determined by their size and speed. They are unstable, and will tend to break up and mix with ambient gas as they rise. Given that they are unlikely to rise faster than the thermal speed, i.e., about $730 \mathrm{~km} \mathrm{~s}^{-1}$, they would take at least $1.3 \times 10^{8} \mathrm{yr}$ to reach $100 \mathrm{kpc}$. This means that some hot bubbles should be present most of the time during the cycles between outbursts. The mass of this hot gas is a few percent of the total gas mass within 100 $\mathrm{kpc}$, so it may be difficult to detect when it is well away from the core. On the other hand, it would be easily detected after the passage of the shock, while it still forms a coherent, overpressured body. Because the hot gas rises relatively quickly (speeds of order the sound speed), it fills a relatively small volume compared to inflowing cooling gas (which is highly subsonic). Thus, apart from some mixing, the outflowing hot bubbles may have relatively little impact on the steady inflow due to cooling.

The shock itself would be easily detectable anywhere within the $30 \mathrm{kpc}$ core region (for $y=2$, the density jump is a factor of 2 and the temperature jump a factor of 1.75). At $r=30 \mathrm{kpc}$, the shock speed is $v_{\mathrm{s}}=\sqrt{3 \gamma k T_{0} / \mu m_{\mathrm{H}}} \simeq 1560 \mathrm{~km} \mathrm{~s}^{-1}$, so that the shock takes about $10^{7} \mathrm{yr}$ to reach $r=30 \mathrm{kpc}$. This means that the shock is easily visible for at least $10 \%$ of the time, or in at least $10 \%$ of all comparable sources. If this model applies to many clusters, such shocks should be detected soon. If shocks are not detected, then shocks from the radio source cannot be responsible for preventing the deposition of cooled gas and we would need to find another heat source.

This description of gas flows around Hydra A presumes that the system remains in a quasi-steady state. Using the relationship between stellar velocity dispersion and the mass of the central black hole (Gebhardt et al. 2000; Ferrarese \& Merrit 2000), the central galaxy in Hydra A should have a nuclear black hole with a mass of $\simeq 5 \times 10^{8} \mathrm{M}_{\odot}$. As cooling gas accumulates in the cluster core, the density of gas around this black hole increases, presumably driving up the accretion rate until there is a radio outburst. The outburst heats and drives away the central gas, thereby reducing the accretion rate and quenching the outburst. This provides a feedback mechanism connecting the average mechanical power of radio outbursts to the total radiative power within the region where there would otherwise be a cooling flow (i.e., where the cooling time is shorter than the age of the system). The critical condition is that the net mass flow 
rate into the core region is kept small (the spectroscopically determined mass deposition rate is less than $10 \%$ of the rate at which cooling gas is delivered to the core in this system). In the absence of a detailed model for the outflow, it is not clear that this requires very close balance between the average mechanical heating rate and radiative cooling. The mechanical heating rate must at least match the cooling rate to prevent accumulation of gas in the core, but any energy deposited outside the cooling flow region has little impact on the current cooling rate and so is not closely controlled by this feedback process. Thus the mechanical heating rate may exceed the radiative heat loss in the cooling flow region. In that case, the net heat input to the cluster could be significant for the cluster as a whole. For example, an average excess heating rate of $10^{45} \mathrm{erg} \mathrm{s}^{-1}$ for $10^{10}$ yr deposited in $10^{14} \mathrm{M}_{\odot}$ of gas amounts to $1.0 \mathrm{keV}$ per particle. This is close to what is required to account for "preheating" in clusters (e.g., Wu, Fabian, \& Nulsen 2000) and the excess heating rate per unit mass could well be greater at early times, when AGNs were more active.

In summary, for Hydra A, gas in the region from about 30 to $150 \mathrm{kpc}$ is cooling steadily and flowing inward, essentially as a (homogeneous) cooling flow at a rate of about $300 \mathrm{M}_{\odot} \mathrm{yr}^{-1}$. Repeated outbursts from the central radio source drive shocks into the cluster and heat the region $r<30 \mathrm{kpc}$ strongly enough to prevent all but about $10 \%$ of the inflowing gas from cooling to low temperatures. Because it is heated from the center, the 30 $\mathrm{kpc}$ core is convective and remains roughly isentropic. During each outburst, gas near the center of the core is heated so much that it rises well outside the core before finding its equilibrium position. On average, the resulting mass outflow nearly balances the cooling inflow to the core. The rate of accretion onto the central black hole that is the source of the radio outbursts is determined by the state of gas in the core. This link provides the feedback that keeps the net gas flow rate into the core close to zero. This model has much in common with the model of Tabor \& Binney (1993) for gas flows in elliptical galaxies. Shock heating fits well with current models of radio outbursts, but if shocks are the main heat source in the core, they soon should be detected in some systems. If shocks are not a major heat source, then any other process by which the AGN heats the cluster from its center would produce a similar general picture. The major factor distinguishing such models is the extent to which the heating is intermittent. Continuous heating would tend to make the whole of the region, which would otherwise be depositing cool gas, isentropic.

\section{SOURCE OF THE CENTRAL ABUNDANCE GRADIENT}

Between 100 and $200 \mathrm{kpc}$ the $\mathrm{Fe}$ and $\mathrm{Si}$ abundances are essentially constant with a $\mathrm{Si} / \mathrm{Fe}$ abundance ratio twice solar (see Figures 10 and 11). This agrees with earlier results from ASCA observations of clusters indicating a significant role of Type II supernovae (SNe II) in the global enrichment of cluster gas (Mushotzky et al. 1996, Fukazawa et al. 1998). Within the central $100 \mathrm{kpc}$ there is an excess of $\mathrm{Si}$ and Fe above the globally averaged values. Using an average $\mathrm{Si}$ abundance of 0.5 solar gives an excess Si mass of $\Delta M_{S i} \simeq 1.4 \times 10^{8} \mathrm{M}_{\odot}$ within the central $100 \mathrm{kpc}$. Using an average $\mathrm{Fe}$ abundance of 0.25 solar gives an excess Fe mass of $\Delta M_{F e} \simeq 3.0 \times 10^{8} \mathrm{M}_{\odot}$ within the same region. The ratio $\Delta M_{S i} / \Delta M_{F e}=0.5$ is essentially consistent with the solar ratio of 0.38 . Fukazawa et al. (2000) and Irwin \& Bregman (2000) find that central metal excesses are common in cD clusters with cooling flows based on ASCA and BEPPOSAX observations. Fukazawa et al. also find that the excess metals have a nearly solar abundance ratio. However, ASCA was unable to resolve the distribution of excess metals in clusters and accurately determine the Fe and Si masses.

The primary sources of metals in the central region of the Hydra A cluster are stellar winds and Type Ia Supernovae (SNe Ia) from the old stellar population in the central galaxy and SNe II from the young stellar population in the blue disk reported by McNamara (1995). A central abundance excess will develop even if the gas is static due to the more extended distribution of the gas compared to the light from the central galaxy. Based on the spectroscopic mass deposition rate, only $10 \%$ of the gas within the central $100 \mathrm{kpc}$ should have condensed out of the hot gas over the cluster lifetime with the remainder being continuously enriched. Convection will flatten the abundance gradients to some extent but will not completely erase all gradients. Convection will also dredge up material from within the central 10 $\mathrm{kpc}$ in the central galaxy that should be enriched by stars with supersolar abundances, typical of the central regions of elliptical galaxies.

To determine the origin of the excess metals, we first compute the number of supernovae required to produce the excess $\mathrm{Si}$ and Fe masses. We adopt the supernovae yields given in Finoguenov, David, \& Ponman (2000), that are averaged over a Salpeter IMF and given by $y_{F e}(I a)=0.74 \mathrm{M}_{\odot}, y_{F e}(I I)=$ $0.070 \mathrm{M}_{\odot}, y_{S i}(I a)=0.158 \mathrm{M}_{\odot}, y_{S i}(I I)=0.133 \mathrm{M}_{\odot}$. The excess Fe mass requires $4 \times 10^{8} \mathrm{SNe} \mathrm{Ia}$. Since the Si yield is nearly independent of supernova type, we can simply assume a yield of $0.14 \mathrm{M}_{\odot}$ per supernovae. To produce the excess Si mass requires $10^{9}$ total supernovae, or $6 \times 10^{8} \mathrm{SNe}$ II. Assuming $1 \mathrm{SNe}$ II for every $100 \mathrm{M}_{\odot}$ of star formation (appropriate for a Salpeter IMF) requires an average star formation rate of $60 \mathrm{M}_{\odot} \mathrm{yr}^{-1}$ over the past $10^{9} \mathrm{yr}$. McNamara (1995) showed that the observed luminosity and colors of the blue disk in Hydra A could only be reproduced by continuous star formation over the past $10^{9} \mathrm{yr}$ with star formation rates of of $\lesssim 1 \mathrm{M}_{\odot} \mathrm{yr}^{-1}$. Higher star formation rates are also permitted by the data, but only when coupled with shorter periods of star formation. It is thus unlikely that the central metal excess in Hydra A was produced by star formation in the blue disk.

The total light of the central galaxy can be estimated from the photometric observations by Peterson (1986) who gives $M_{V}=$ -23.65 and $\mathrm{B}-\mathrm{V}=1.08$ within a $1.65^{\prime}$ diameter aperture. This gives $L_{B}=9.2 \times 10^{10} L_{\odot}$ within the central $47 \mathrm{kpc}$ radius using our adopted cosmology. To reproduce the observed excess Fe mass requires an average $\mathrm{SNe}$ Ia rate of $4 \times 10^{-13} L_{B}^{-1} \mathrm{yr}^{-1}$ over the past $10^{10}$ years, which is approximately twice Tammann's (1974) rate of $2.2 \times 10^{-13} L_{B}^{-1} \mathrm{yr}^{-1}$. Tammann's rate is usually considered to be a factor of 4 too high (van den Bergh, McClure, \& Evans 1987), but our estimate does not include the contribution from stars beyond the central $50 \mathrm{kpc}$. Given the large uncertainty in the present epoch $\mathrm{SNe}$ Ia rate in elliptical galaxies, the excess Fe can be accounted for by SNe Ia from the old stellar population of the central galaxy.

Mass loss from stars as they evolve off the main sequence also can be a significant source of metals. Using the Faber \& Gallagher (1976) mass loss rate of $10^{-11} \mathrm{M}_{\odot} \mathrm{L}_{\mathrm{V}}^{-1} \mathrm{yr}^{-1}$ gives a stellar mass loss rate of $2.5 \mathrm{M}_{\odot} \mathrm{yr}^{-1}$ within the central $47 \mathrm{kpc}$. If this gas has a solar abundance of heavy elements, and the present mass loss rate is assumed to hold over the lifetime of the galaxy, then stellar mass loss can only produce about $15 \%$ of the observed $\mathrm{Si}$ and Fe excess, and only about $6 \%$ of the total gas mass within this radius. 
There is considerable evidence that the environment of earlytype galaxies has a significant impact on their X-ray properties (e.g., Brown \& Bregman 1998; Helsdon et al. 2000), with isolated ellipticals having the lowest $L_{x} / L_{B}$ ratios and the central dominant ellipticals in groups having the highest $L_{x} / L_{B}$ ratios. Brighenti \& Mathews (1998) have suggested that much of the hot gas in ellipticals arises from the accretion of circumgalactic gas. While the central galaxy in Hydra A is a rather extreme example, since it is located at the center of a rich cluster, it is useful to compare the gaseous properties within the central $10 \mathrm{kpc}$ with that found in samples of more isolated ellipticals. The deprojected X-ray luminosity of the hot gas within the central $10 \mathrm{kpc}$ is $L(0.5-2.0 \mathrm{keV})=8.6 \times 10^{42} \mathrm{ergs} \mathrm{s}^{-1}$ (excluding the central point source). Compared with the samples of ellipticals in Brown \& Bregman and Helsdon et al., the central galaxy in Hydra A is 100 times overluminous in X-rays for its observed $L_{B}$. The deprojected gas temperature within the central $10 \mathrm{kpc}$ is $2.7 \mathrm{keV}$ which is approximately 3 times higher than a typical elliptical. The higher temperature cannot result from heating by supernovae. Based on the calculations above, the production of the excess Si mass via SNe would only heat the gas by $0.6 \mathrm{keV}$ per particle. Thus, the higher gas temperature must result from gravitational infall and compression. This is consistent with the result noted above that the hot gas in the central galaxy cannot be produced by stellar mass loss and must originate from the intracluster medium.

\section{SUMMARY}

We have presented a detailed analysis of the Chandra observations of the Hydra A cluster. The high spatial resolution of Chandra and the good photon statistics in these observations have allowed us to study the distribution of gas temperature, multiphase gas, and metals in the cluster core on scales of 10$20 \mathrm{kpc}$. These high resolution observations present significant challenges to the conventional cooling flow scenario and the processes by which the hot gas in clusters is enriched with heavy elements.

The large scale abundance ratio of heavy elements in the Hydra A cluster determined from the Chandra observations indicates that the gas was initially enriched by a predominance of SNe II, in agreement with earlier ASCA observations. The uniform distribution of $\mathrm{Fe}$ and $\mathrm{Si}$ beyond the central $100 \mathrm{kpc}$ suggests that this enrichment occurred prior to the last major merger, during which any pre-existing gradients would have been erased. Within the central $100 \mathrm{kpc}$, the observed excess of $\mathrm{Fe}$ and $\mathrm{Si}$ has a solar abundance ratio.

The ACIS-S spectra do not show any statistically significant evidence for multiphase gas beyond the central $30 \mathrm{kpc}$, even through the cooling time of the gas at this radius is only 1 Gyr. Within $30 \mathrm{kpc}$, the spectroscopic mass deposition rate is roughly consistent with the observed star formation rate, but is more than an order of magnitude less than the morphological mass accretion rate at the same radius. The gas is also isentropic within the central $30 \mathrm{kpc}$. Based on these observations, we propose a scenario in which a small amount of the hot gas is able to cool and accrete onto the central black hole in Hydra A and trigger the formation of a radio jet which mechanically heats the gas via strong shocks. We show that the collective effect of numerous weak shocks is energetically insufficient to have a major impact on the cooling flow. The boost in gas entropy after the passage of a strong shock makes the central gas convectively unstable and produces buoyant bubbles of hot gas. Averaged over many outbursts, the mass accretion rate in the outer cooling flow region can be nearly balanced by the mass outflow rate in the expanding bubbles. This scenario makes definitive predictions that can be tested in the near future with Chandra and XMM. About $10 \%$ of cooling flow clusters should show evidence of strong shocks. By the end of AO-2, Chandra will have observed approximately 100 groups and clusters so this prediction can be easily tested. In addition, the greater throughput of XMM-Newton may permit the spectroscopic detection of hot buoyant bubbles in the outer parts of cooling flows. Based on our results, it is very likely that some there will be some fundamental changes in the cooling flow scenario over the next few years as data from Chandra and XMM-Newton continue to accumulate.

PEJN and TJP gratefully acknowledge the hospitality of the Harvard-Smithsonian Center for Astrophysics. We are grateful to J. Mohr for a very informative discussion about recent theoretical and observational results concerning dark matter halos. This work was partially supported by NASA contract NAS839073.

\section{REFERENCES}

Brighenti, F. \& Mathews, W.G. 1998, ApJ, 495, 239.

Broadhurst, T., Huang, X., Frye, B., \& Ellis, R. 2000, ApJ, 534, 15.

Brown, B.A. \& Bregman, L.N. 1998, ApJ, 495, L75.

Burkert, A. 1995, ApJ, 447, L25.

David, L.P., Arnaud, K., Forman, W., \& Jones C. 1990, ApJ, $356,32$.

Donahue, M., Mack, J., Voit, G.M., Sparks, W., Elston, R., \& Maloney, P.R. 2000, (astro-ph 0007062).

Evrard, A.E. 1997, MNRAS, 292, 289.

Faber, S.M. \& Gallagher, J.S. 1976, ApJ, 204, 365.

Fabian, A.C. \& Nulsen P.E.J. 1977, MNRAS, 180, 479.

Fabian, A.C. 1994, ARAA, 32, 277.

Fabian, A.C., Sanders, J., Ettori, S., Taylor, G., Allen, S., Crawford, C., Iwasawa, K., Johnstone, R. \& Ogle, P. 2000, (astro-ph 0007456).

Ferrarese, L. \& Merrit, D. 2000, ApJ, 539, L9.

Finoguenov, A., David, L., Ponman, T. 2000 (preprint).

Flores, R. \& Primack, J. 1994, ApJ, 427, L1.

Fukazawa, Y., Makishima, K., Tamura, T., Ezawa, H., Xu, H., Ikebe, Y., Kikuchi, K., Ohashi, T. 1998, PASJ, 50, 187.

Fukazawa, Y., Makishima, K., Tamura, T., Nakazawa, K., Ezawa, H., Ikebe, Y., Kikuchi, K., Ohashi, T. 2000, MNRAS, 313, 21.

Gebhardt, K., Bender, R., Bower, G., Dressler, A., Faber, S., Filippenko, A., Green, R., Grillmair, C., Ho, L., Kormendy, J., Lauer, T., Magorrian, J., Pinkney, J., Richstone, D., \& Tremaine, S. 2000, ApJ, 539, L13.

Heckman, T. M., Baum, S.A., van Breugel, \& W., McCarthy, P. 1989, Ap.J., 338, 48.

Helsdon, S.F., Ponman, T.J., O'Sullivan, E., \& Forbes, D.A. 2000 (preprint).

Heinz, S., Reynolds, C. \& Begelman, M. 1998, ApJ, 501, 126.

Hu, E.M., Cowie, L.L., \& Wang, Z. 1985, Ap.J. Suppl., 59, 447.

Ikebe, Y., Makishima, K., Ezawa, H., Fukazawa, Y., Hirayama, M., Honda H., Ishisake, Y., Kikuchi, K., Kubo, H., Murakami, T., Ohashi, T., Takahashi, T., \& Yamashita, K. 1997, ApJ, 481, 660. 
Irwin, J.A. \& Bregman, J.N. 2000 (astro-ph 0009237).

Jing, Y. 2000, ApJ, 535, 30.

Lewis, G., Babul, A., Katz, N., Quinn, T., Hernquist, L., \& Weinberg, D. 2000, ApJ, 536, 623

Markevitch, M., Forman, W., Sarazin, C., \& Vikhlinin, A. 1998, ApJ, 503, 77.

McElroy, D.B. 1995, A\&A Supp, 100, 105.

McGaugh, S. \& de Block, W. 1998, ApJ, 499, 41.

McNamara, B., \& O’Connell, R. 1992, ApJ., 393, 579.

McNamara, B. 1995, ApJ, 443, 77.

McNamara, B., Wise, M., Nulsen, P. E. J., David, L., Sarazin, C., Bautz, M., Markevitch, M., Vikhlinin, A., Forman, W., Jones, C., Harris, D. 2000, ApJ, 534, 135L.

Melnick, J., Gopal-Krishna, \& Terlevich, R. 1997, AA, 318, 337.

Mohr, J., Mathiesen, B., \& Evrard. A. 1999, ApJ, 517, 627.

Moore, B., Quinn, T., Governato, F., Stadel, J., \& Lake, G. 1999, MNRAS, 310, 1147.

Mushotzky, R.F., Loewenstein, M., Arnaud, K.A., Tamura, T., Fukazawa, Y., Matsushita, K., Kikuchi, K., Hatsukade, I. 1996, ApJ, 466, 686.

Navarro, J., Frenk, C., \& White, S. 1995, MNRAS, 275, 720.

Navarro, J., Frenk, C., \& White, S. 1997, ApJ, 490, 493.

Peres, C., Fabian, A., Edge, A., Allen, A., Johnstone, R., \& White, D. 1998, MNRAS, 298, 416.

Peterson, C.J. 1986, PASP, 98, 486.

Robertson, B. \& David, L. 2000 (in preparation).

Sambruna, R., Chartas, G., Eracleous, M., \& Mushotzky, R. 2000, (astro-ph 0002201).

Sarazin, C.S. 1986, Rev. Mod. Phys., 58, 1.

Shapiro, P. \& Iliev, I. 2000 (astro-ph 0006353).

Soker, N., White, R., David, L. \& McNamara, B 2000 (astro-ph 0009173).

Swaters, R., Madore, B., \& Trewhella, M. 2000, ApJ, 531, L107.

Tammann, G.A. 1974, Supernovae and Their Remnants, ed. C.B. Comsovici (Dordrecht:Reidel), p. 155.

Taylor, G.B. 1996, ApJ, 470, 394.

Thomas, P.A., Fabian, A.C., \& Nulsen, P.E.J. 1987, MNRAS, 228, 973.

Tucker, W. \& David, L. 1997, ApJ, 484, 602.

Tyson, J.A., Kochanski, G. \& Dell'Antonio, I. 1998, ApJ, 498, L107.

van den Bergh, S., McClure, R.D., \& Evans, R. 1987, ApJ, 323, 44.

van den Bosch, F., Robertson, B., Dalcanton, J. \& de Blok, W. 2000, AJ, 119, 1579.

Vikhlinin, A., Forman, W., Jones, C. 1999, ApJ, 525, 47.

Weisskopf, M., Tananbaum, H., Van Speybroeck, L., \& O’Dell, S. 2000, Proc SPIE 4012 (astro-ph 0004127).

White, D. \& Fabian, A.C. 1995, MNRAS, 273, 72.

White, D. 2000, MNRAS, 312, 663.

Wilson, A.S., Young, A.J. \& Shopbell, P.L. 2000, (astro-ph 0009308) 
TABLE 1

Comparison of Spectral Fits for the $2^{\prime \prime}-17^{\prime \prime}$ annulus

\begin{tabular}{|c|c|c|c|c|c|c|c|}
\hline Model & $\begin{array}{c}\mathrm{kT} \\
(\mathrm{keV})\end{array}$ & $\begin{array}{c}N_{H} \\
\left(10^{20} \mathrm{~cm}^{-2}\right)\end{array}$ & $\begin{array}{c}\mathrm{Z} \\
\text { (solar) }\end{array}$ & $\begin{array}{c}\dot{\mathrm{M}} \\
\left(\mathrm{M}_{\odot} \mathrm{yr}^{-1}\right)\end{array}$ & $\begin{array}{c}N_{H i} \\
\left(10^{20} \mathrm{~cm}^{-2}\right)\end{array}$ & $\begin{array}{l}k T_{\text {low }} \\
(\mathrm{keV})\end{array}$ & $\chi^{2} / \mathrm{DOF}$ \\
\hline \multirow[t]{2}{*}{ WABS*MEKAL } & $2.78_{-09}^{+.08}$ & 4.88 & $0.36_{-05}^{+.06}$ & - & - & - & $250 / 181$ \\
\hline & $2.88_{-.11}^{+.18}$ & $3.65_{-.80}^{+.79}$ & $0.41_{-.06}^{+.07}$ & - & - & - & $242 / 180$ \\
\hline WABS*(MEKAL & $3.00_{-.13}^{+.15}$ & 4.88 & $0.44_{-.06}^{+.06}$ & $8.9_{-4.9}^{+4.1}$ & - & - & $242 / 180$ \\
\hline + MKCFLOW) & $3.11_{-.15}^{+.14}$ & $3.63_{-.68}^{+.57}$ & $0.49_{-.07}^{+.07}$ & $6.3_{-4.3}^{+4.3}$ & - & - & $237 / 179$ \\
\hline WABS*(MEKAL & $3.05_{-.17}^{+.11}$ & 4.88 & $0.44_{-.06}^{+.06}$ & $8.7_{-3.9}^{+4.3}$ & $<3.2$ & - & $241 / 179$ \\
\hline +ZWABS*MKCFLOW) & $3.10_{-.15}^{+.14}$ & $<2.3$ & $0.48_{-.06}^{+.08}$ & $29_{-17}^{+12}$ & $38.0_{-3.9}^{+4.3}$ & - & $232 / 178$ \\
\hline WABS*(MEKAL & $4.08_{-1.02}^{+3.9}$ & 4.88 & $0.31_{-.05}^{+.06}$ & - & - & $1.93_{-.32}^{+.68}$ & $240 / 179$ \\
\hline +MEKAL) & $3.72_{-.90}^{+2.4}$ & $3.56_{-0.5}^{+.80}$ & $0.37_{-.07}^{+.06}$ & - & - & $1.91_{-.72}^{+.59}$ & $233 / 178$ \\
\hline
\end{tabular}

Notes: The results of fitting the ACIS-S data to a variety of spectral models (defined in column 1). $k T$ is the gas temperature in single temperature fits and the highest temperature in two-temperature fits, $N_{H}$ is the hydrogen column density, $Z$ is abundance of heavy elements relative to the solar value, $\dot{M}$ is the mass deposition rate in models with a cooling flow component, and $k T_{l o w}$ is the lower gas temperature in the two-temperature fit. When fitting the single temperature plus cooling flow model, the abundance of heavy elements is linked between the two components and the lowest gas temperature is fixed at 0.02 keV. Two rows are shown for each model. The top row shows the results with $N_{H}$ fixed to the galactic value and the second row shows with results with $N_{H}$ free. All error bars are shown at the $90 \%$ confidence level for one interesting parameter $\left(\chi_{\min }^{2}+2.71\right)$.

TABLE 2

Comparison of Spectral Fits for the $17^{\prime \prime}-29^{\prime \prime}$ annulus

\begin{tabular}{c|cccccc}
\hline \hline Model & $\begin{array}{c}\mathrm{kT} \\
(\mathrm{keV})\end{array}$ & $\begin{array}{c}N_{H} \\
\left(10^{20} \mathrm{~cm}^{-2}\right)\end{array}$ & $\begin{array}{c}\mathrm{Z} \\
(\mathrm{solar})\end{array}$ & $\begin{array}{c}\dot{\mathrm{M}} \\
\left(\mathrm{M}_{\odot} \mathrm{yr}^{-1}\right)\end{array}$ & $\begin{array}{c}N_{H i} \\
\left(10^{20} \mathrm{~cm}^{-2}\right)\end{array}$ & $\chi^{2} / \mathrm{DOF}$ \\
\hline WABS*MEKAL & $2.97_{-.13}^{+.11}$ & 4.88 & $0.35_{-.05}^{+.06}$ & - & - & $249 / 190$ \\
& $3.15_{-.15}^{+.14}$ & $3.44_{-.67}^{+.66}$ & $0.41_{-.06}^{+.06}$ & - & - & $236 / 189$ \\
& & & & & & $244 / 189$ \\
WABS*(MEKAL & $3.15_{-.17}^{+.17}$ & 4.88 & $0.38_{-.05}^{+.06}$ & $7.6_{-4.2}^{+4.3}$ & - & $236 / 188$ \\
+ MKCFLOW) & $3.24_{-.17}^{+.18}$ & $3.65_{-.70}^{+.72}$ & $0.42_{-.06}^{+.07}$ & $4.8_{-4.4}^{+4.4}$ & - & $244 / 188$ \\
WABS*(MEKAL & $3.15_{-.17}^{+.17}$ & 4.88 & $0.38_{-.05}^{+.06}$ & $7.4_{-3.2}^{+4.5}$ & $<3.1$ & $236 / 187$ \\
\hline
\end{tabular}

Notes: see the notes for Table 1 .

TABLE 3

Comparison of Spectral Fits for the $29^{\prime \prime}-43^{\prime \prime}$ annulus

\begin{tabular}{c|ccccc}
\hline \hline Model & $\begin{array}{c}\mathrm{kT} \\
(\mathrm{keV})\end{array}$ & $\begin{array}{c}N_{H} \\
\left(10^{20} \mathrm{~cm}^{-2}\right)\end{array}$ & $\begin{array}{c}\mathrm{Z} \\
(\mathrm{solar})\end{array}$ & $\begin{array}{c}\dot{\mathrm{M}} \\
\left(\mathrm{M}_{\odot} \mathrm{yr}^{-1}\right)\end{array}$ & $\chi^{2} / \mathrm{DOF}$ \\
\hline WABS*MEKAL & $3.25_{-.12}^{+.12}$ & 4.88 & $0.34_{-.05}^{+.06}$ & - & $259 / 232$ \\
& $3.36_{-.15}^{+.14}$ & $4.03_{-.67}^{+.70}$ & $0.38_{-.06}^{+.06}$ & - & $255 / 231$ \\
& & & & & \\
WABS*(MEKAL & $3.39_{-.17}^{+.18}$ & 4.88 & $0.36_{-.05}^{+.06}$ & $<9.9$ & $256 / 231$ \\
+ MKCFLOW) & $3.43_{-.17}^{+.18}$ & $4.22_{-.71}^{+.72}$ & $0.38_{-.06}^{+.07}$ & $<8.1$ & $254 / 230$ \\
\hline
\end{tabular}

Notes: see the notes for Table 1. 


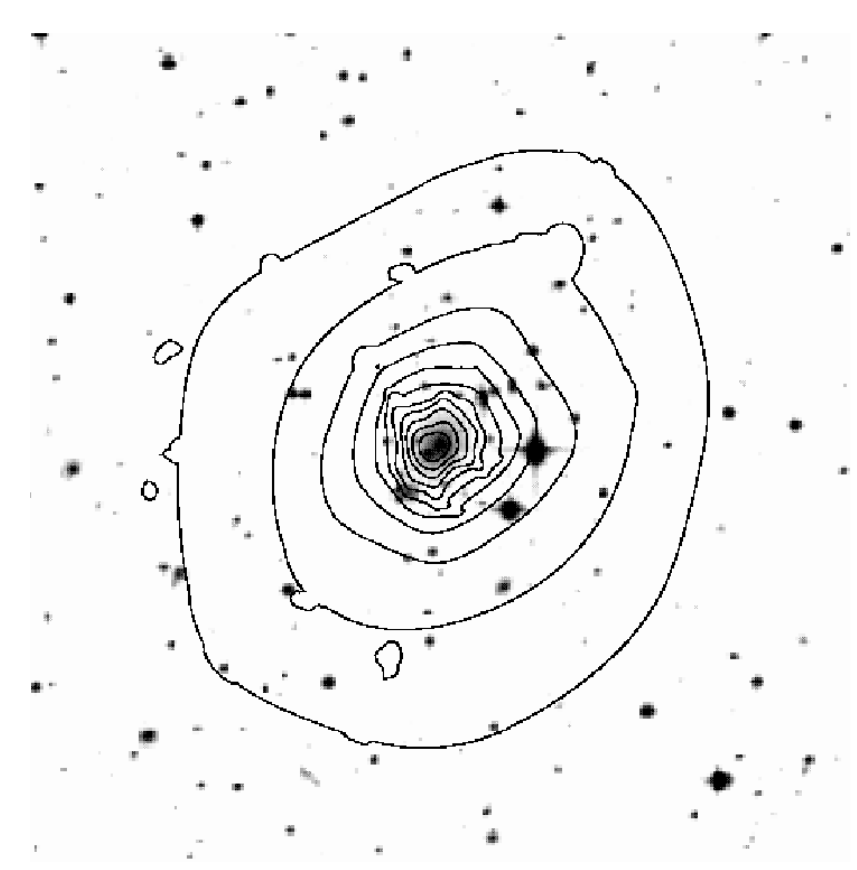

FIG. 1. ACIS-I contours of the background subtracted, vignetting and exposure corrected ACIS-I data overlaid on the optical image of the Hydra A cluster. The figure is $8.53^{\prime}(540 \mathrm{kpc})$ on a side.

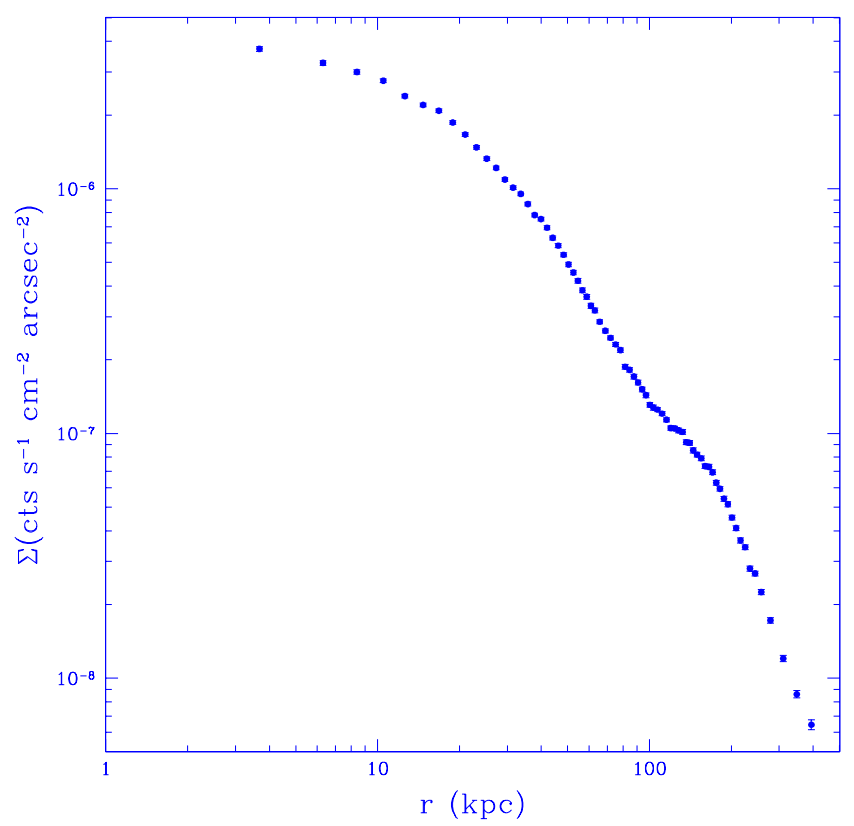

FIG. 2. The 0.3-7.0 keV surface brightness profile of the Hydra A cluster from the ACIS-S observation. The data are background subtracted, vignetting and exposure corrected, and binned to produce 2000 net counts per annulus. 


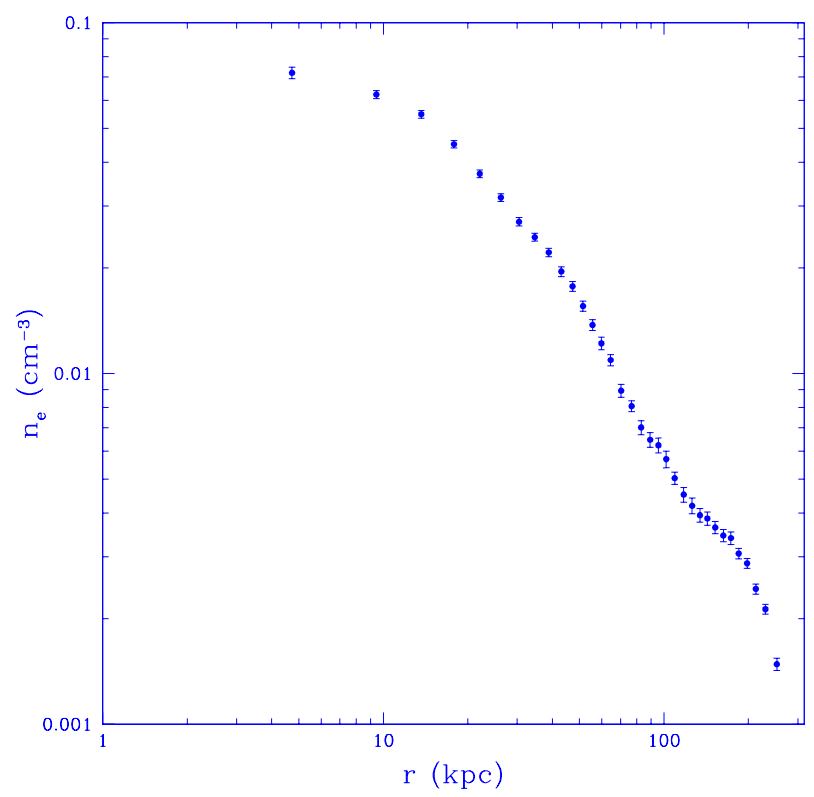

FIG. 3. The deprojected electron density profile of the Hydra A cluster with errors derived from 100 Monte Carlo simulations.

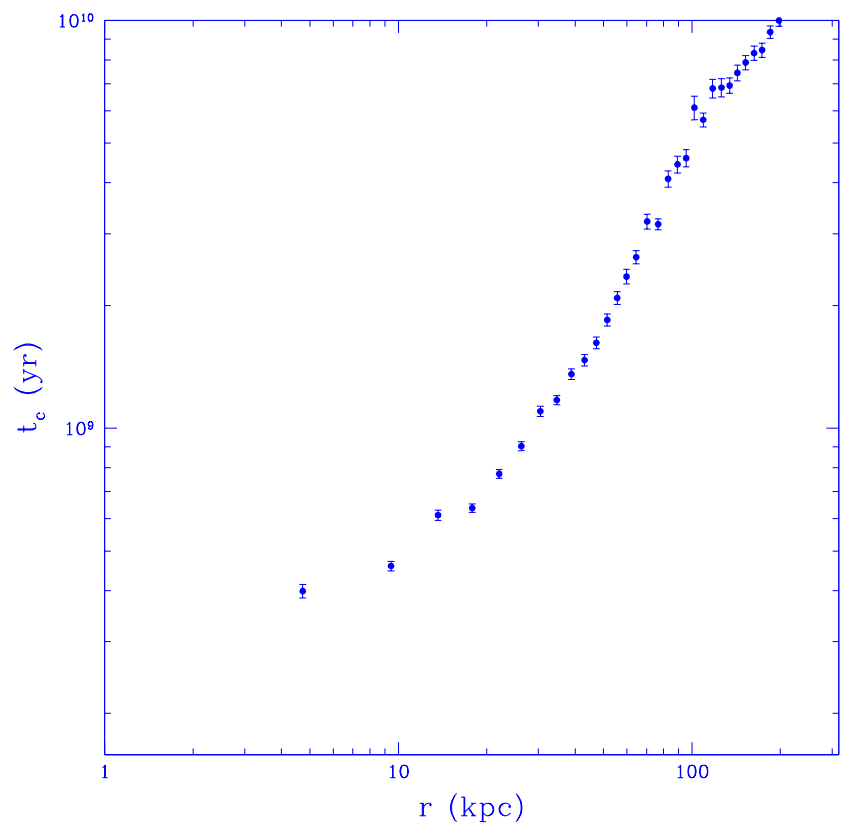

FIG. 4. The isobaric cooling time of the hot gas as a function of radius. 

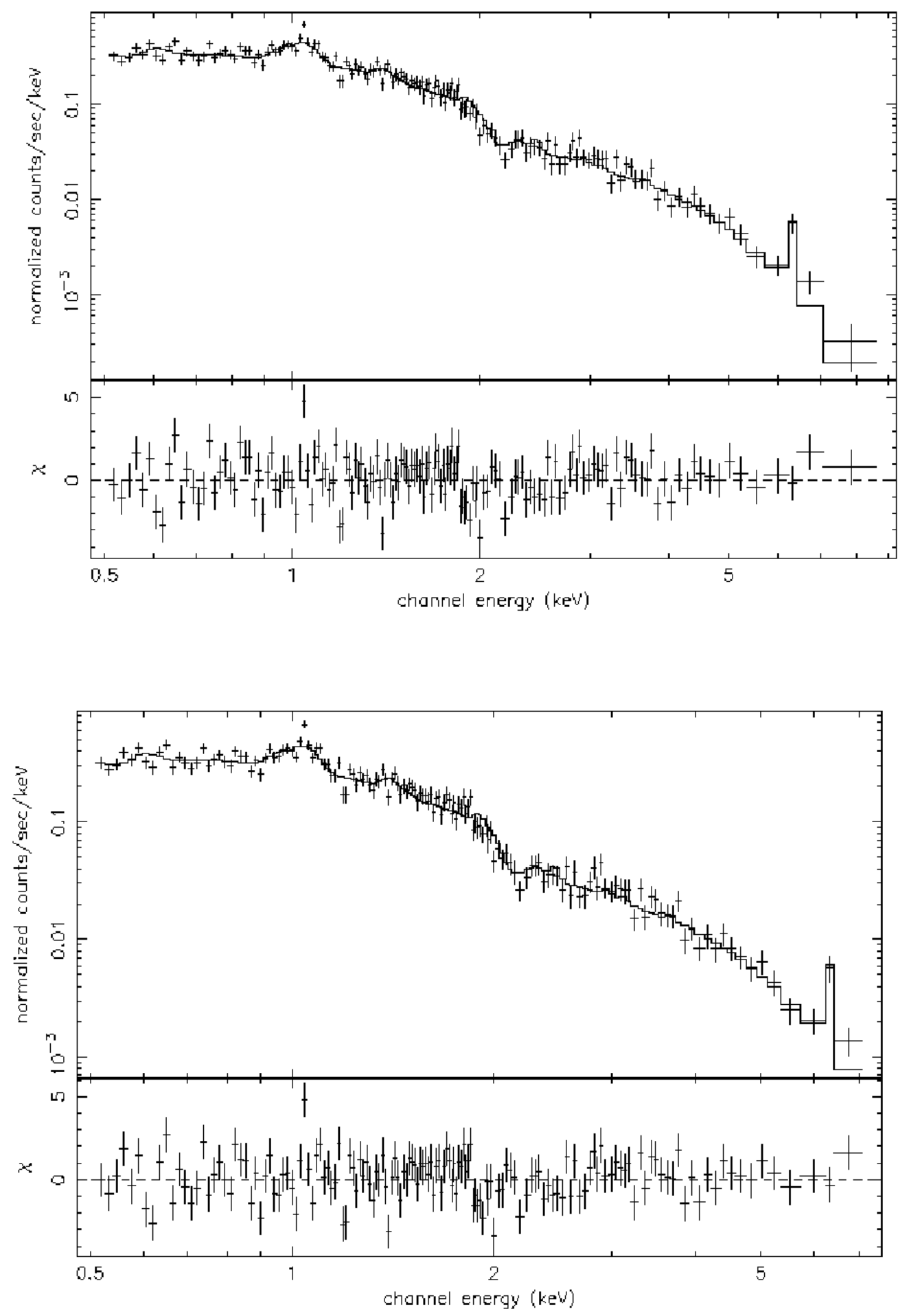

FIG. 5. a) Best-fit single temperature model to the innermost ACIS-S spectrum $\left(2^{\prime \prime}-17^{\prime \prime}\right)$, and b) best-fit single temperature plus cooling flow model to the innermost ACIS-S spectrum. 


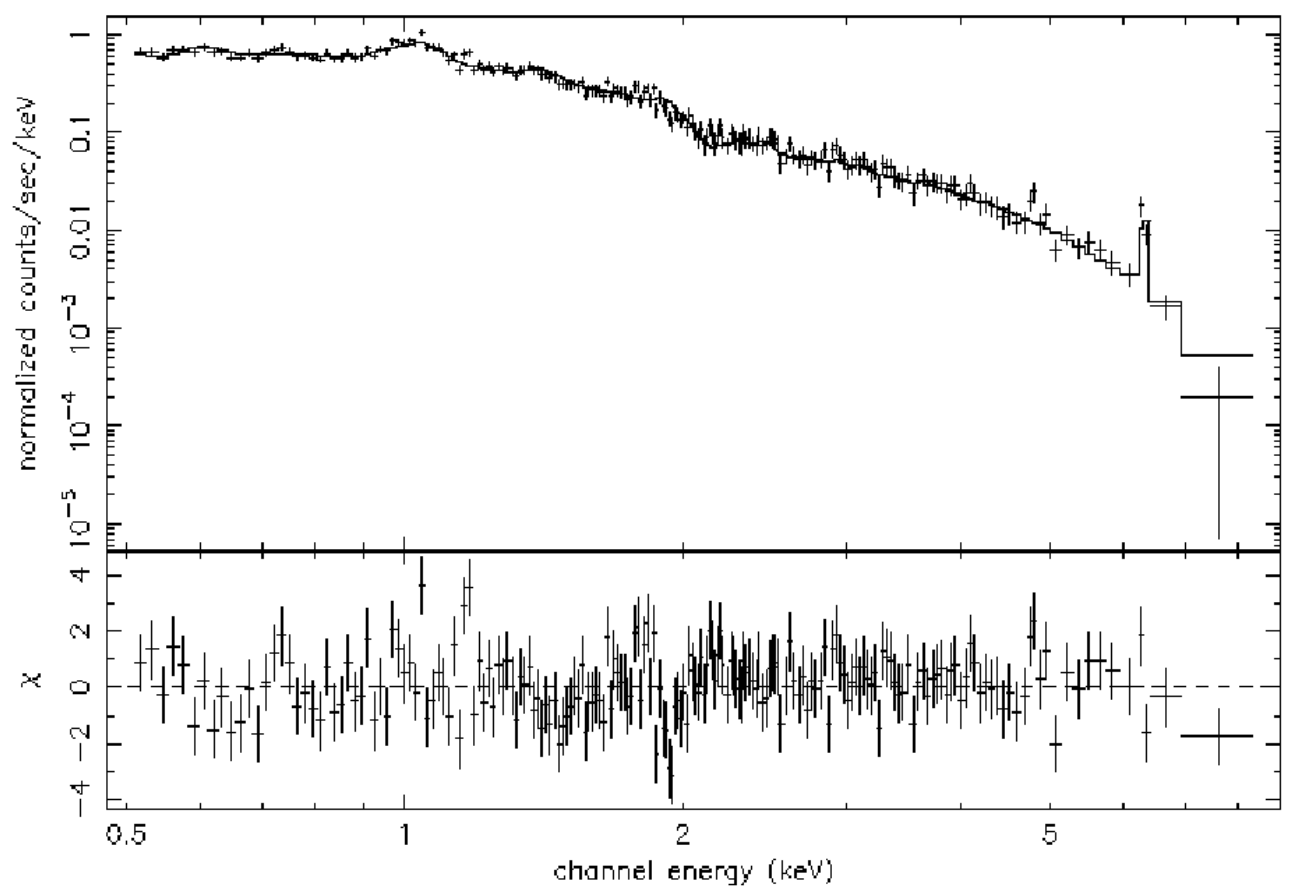

FIG. 6. Best-fit single temperature model to the ACIS-S spectrum extracted from an annulus between $17^{\prime \prime}$ and $29^{\prime \prime}$ from the central point source.

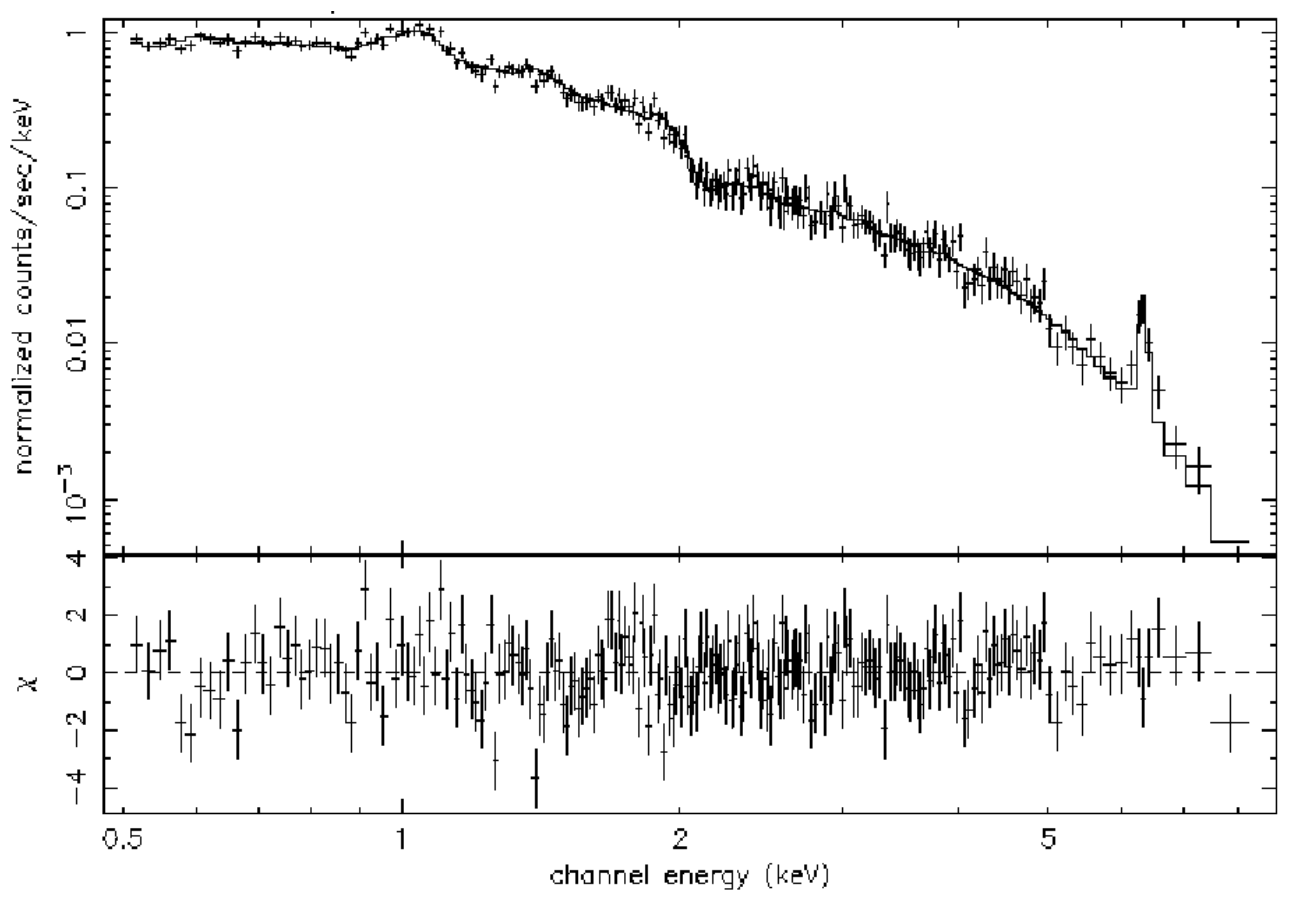

FIG. 7. Best-fit single temperature model to the ACIS-S spectrum extracted from an annulus between $29^{\prime \prime}$ and $43^{\prime \prime}$ from the central point source. 


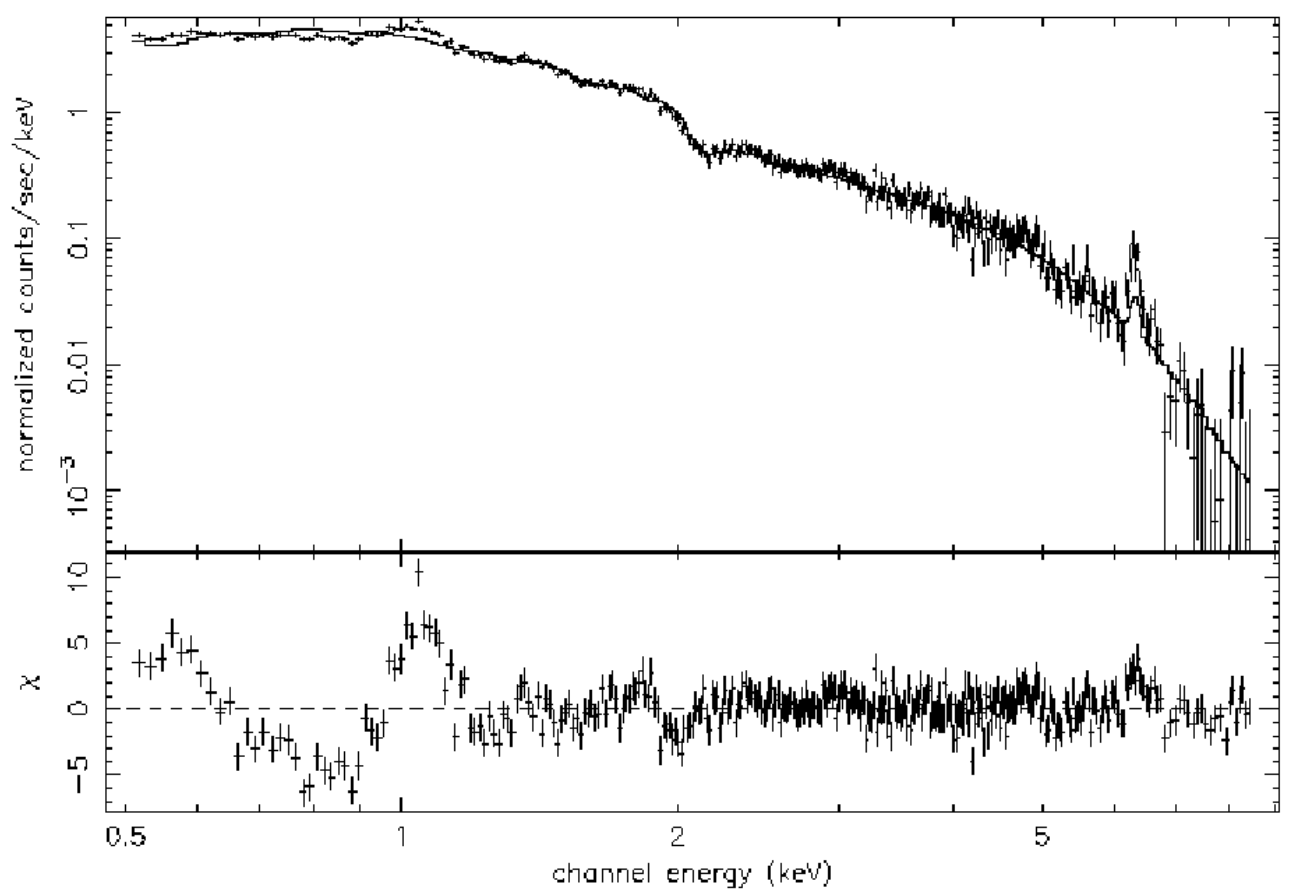

FIG. 8. Best-fit of the ACIS-S spectrum of the central $100 \mathrm{kpc}$ (excluding the central point source) to a single temperature plus cooling flow model with $\dot{\mathrm{M}}$ frozen at $140 \mathrm{M}_{\odot} \mathrm{yr}^{-1}$ and all other parameters free. 


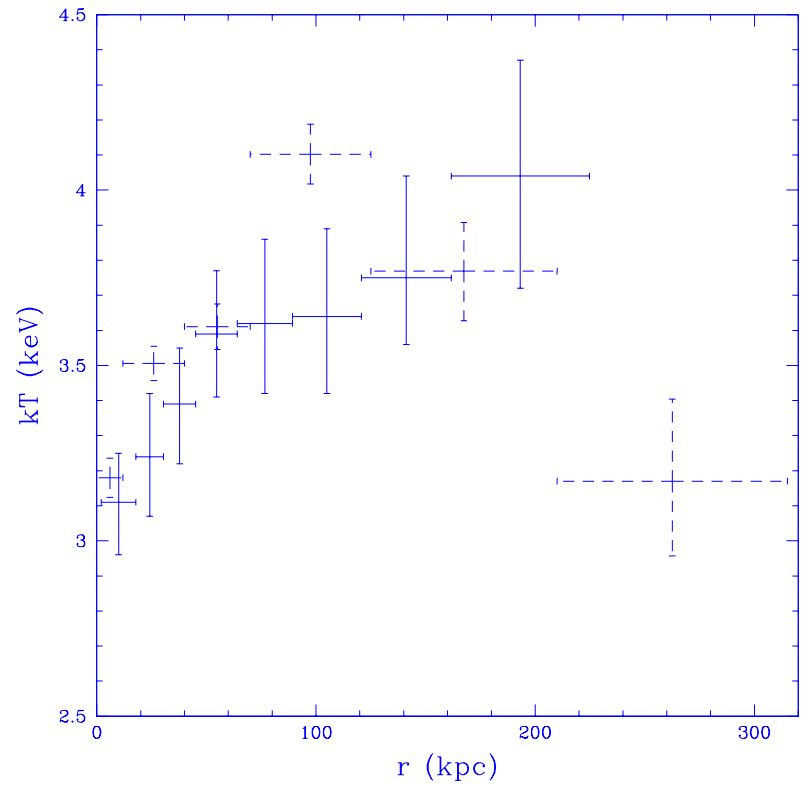

FIG. 9. Gas temperature profile of the Hydra A cluster. The solid (dashed) crosses show the spectral analysis results of the ACIS-S (ACIS-I) data. Error bars are shown at the $90 \%$ confidence level for one interesting parameter.

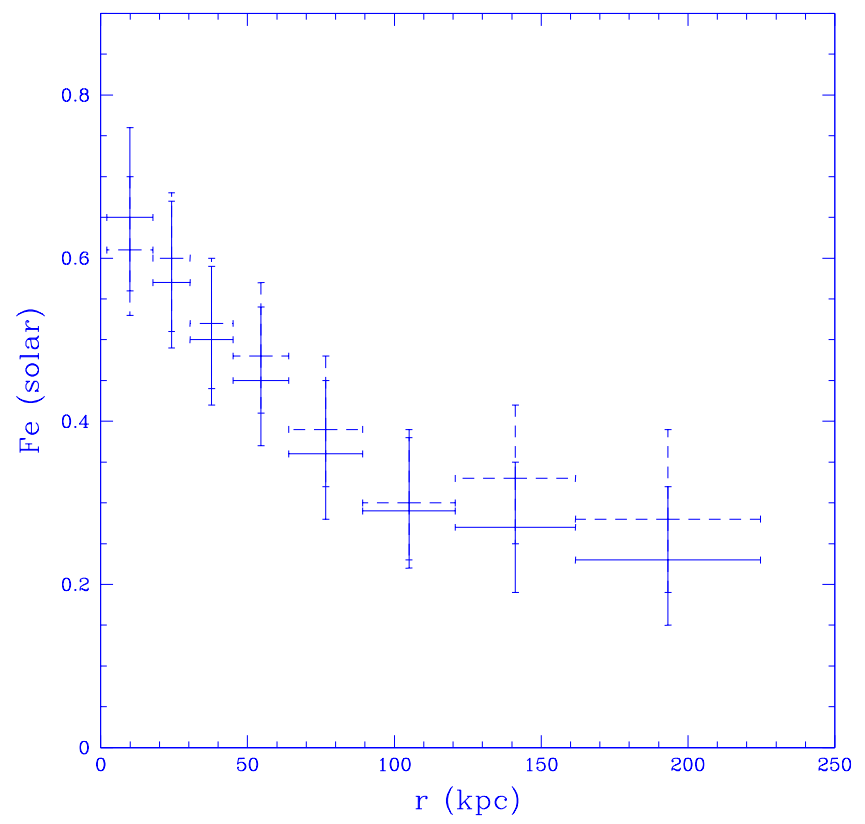

FIG. 10. Fe abundance profile relative to the solar value $\left(4.68 \times 10^{-5}\right.$ by number relative to $\left.\mathrm{H}\right)$ for the Hydra A cluster derived from the ACIS-S observation. The solid lines show the best fit to a single temperature model in the $0.5-7.5 \mathrm{keV}$ band, while the dashed lines show the the best fit to a single temperature model in the $2.0-7.5 \mathrm{keV}$ band. Error bars are shown at the $90 \%$ confidence level for one interesting parameter. 


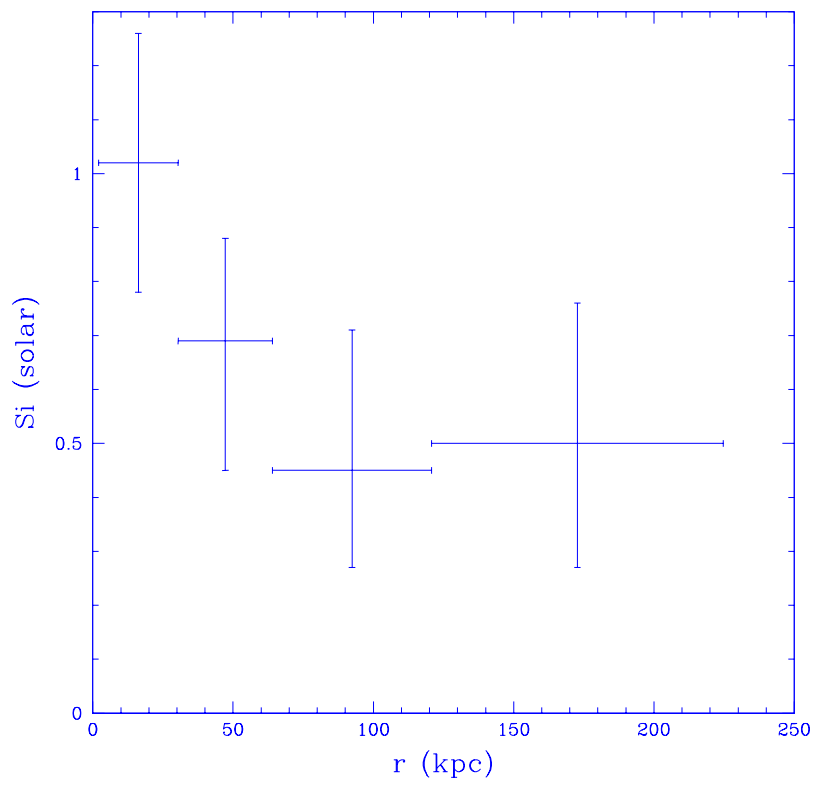

FIG. 11. Si abundance profile relative to the solar value $\left(3.55 \times 10^{-5}\right.$ by number relative to $\left.\mathrm{H}\right)$ for the Hydra A cluster derived from the ACIS-S observation. Error bars are shown at the $90 \%$ confidence level for one interesting parameter.

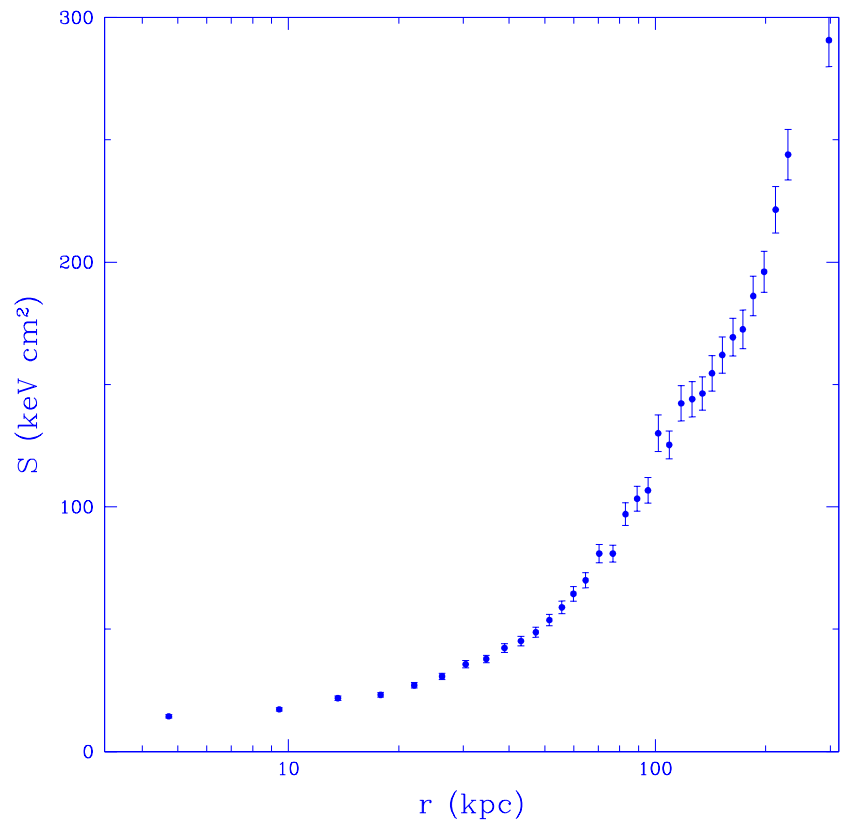

FIG. 12. Gas entropy profile derived from the deprojected density and power-law temperature profiles. 


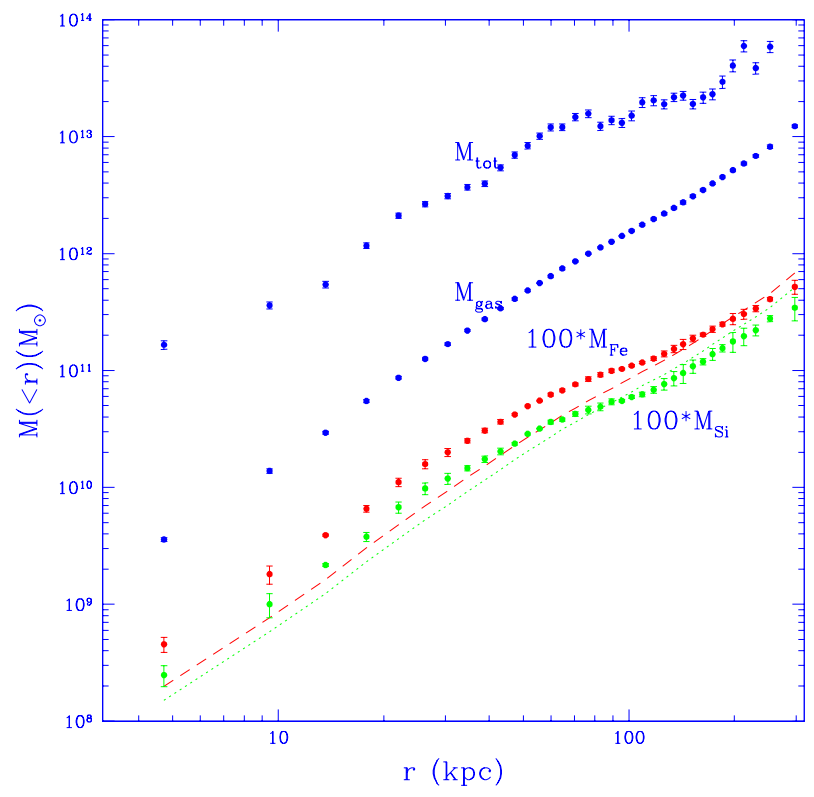

FIG. 13. The integrated total gravitating mass, gas mass, Fe mass, and $\mathrm{Si}$ mass distributions. The Fe and $\mathrm{Si}$ masses are shown at 100 times their true values. The dashed and dotted lines are the Fe and Si masses that would be inferred assuming a uniform distribution of heavy elements.

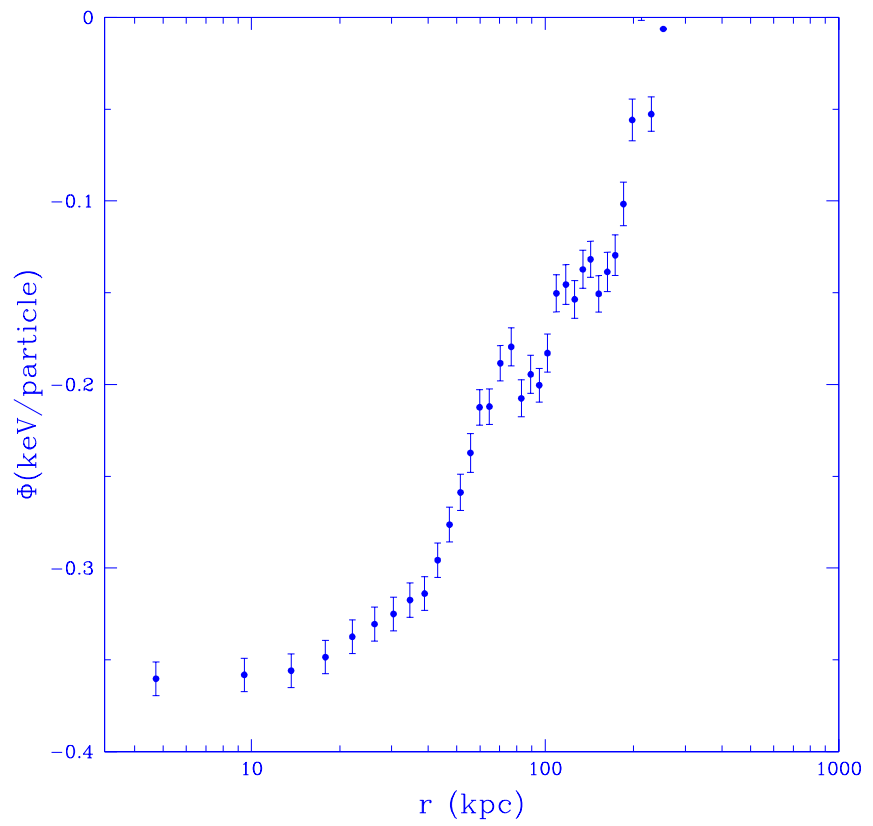

FIG. 14. The gravitational potential in the Hydra A cluster derived from the total mass distribution shown in Figure 8 . The potential is normalized to zero at $250 \mathrm{kpc}$. 


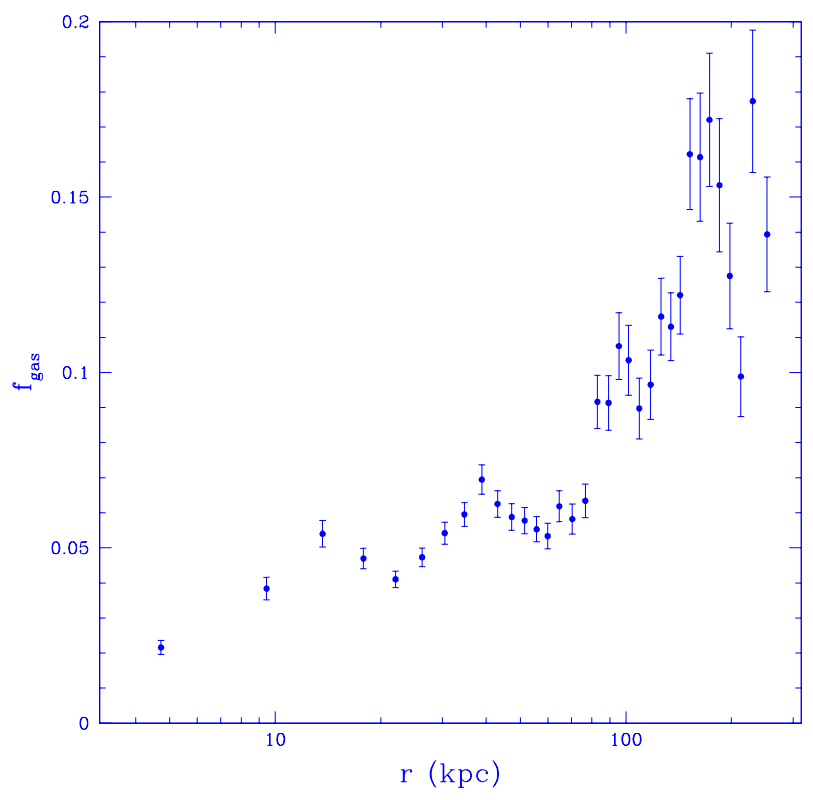

FIG. 15. Gas mass fraction versus radius in the Hydra A cluster.

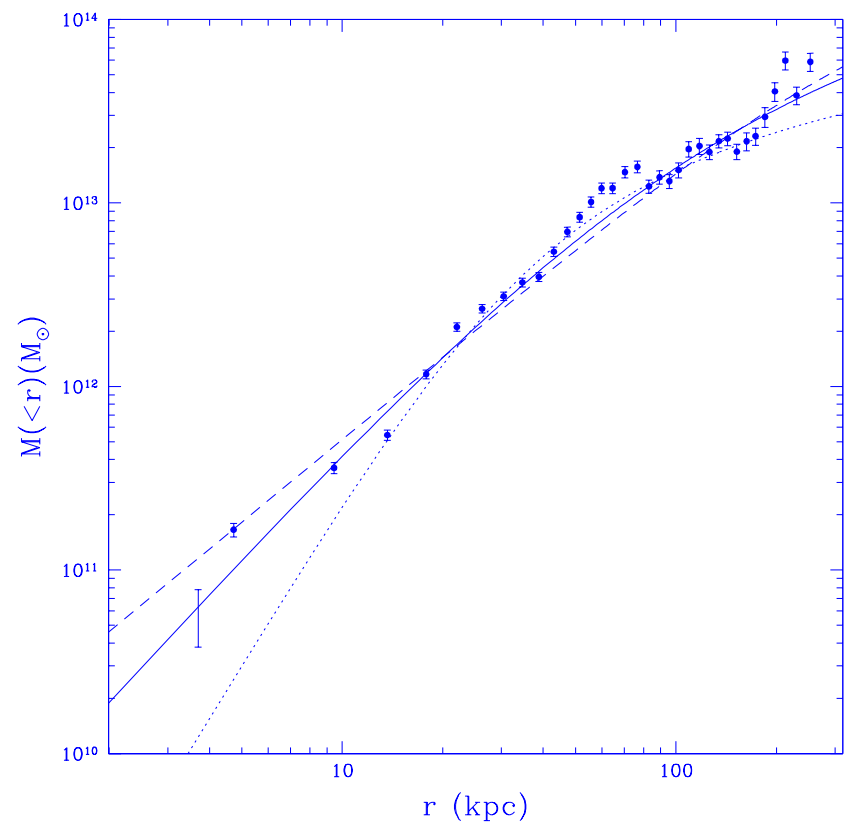

FIG. 16. Comparison of the best-fit NFW profile (solid line), Moore profile (dashed line), and modified King profile (dotted line) to the cumulative gravitating mass distribution in Hydra A. The point at $3.7 \mathrm{kpc}$ is the mass inferred from the rotation curve of the $\mathrm{H} \alpha$ disk (Melnick et al. 1997). 


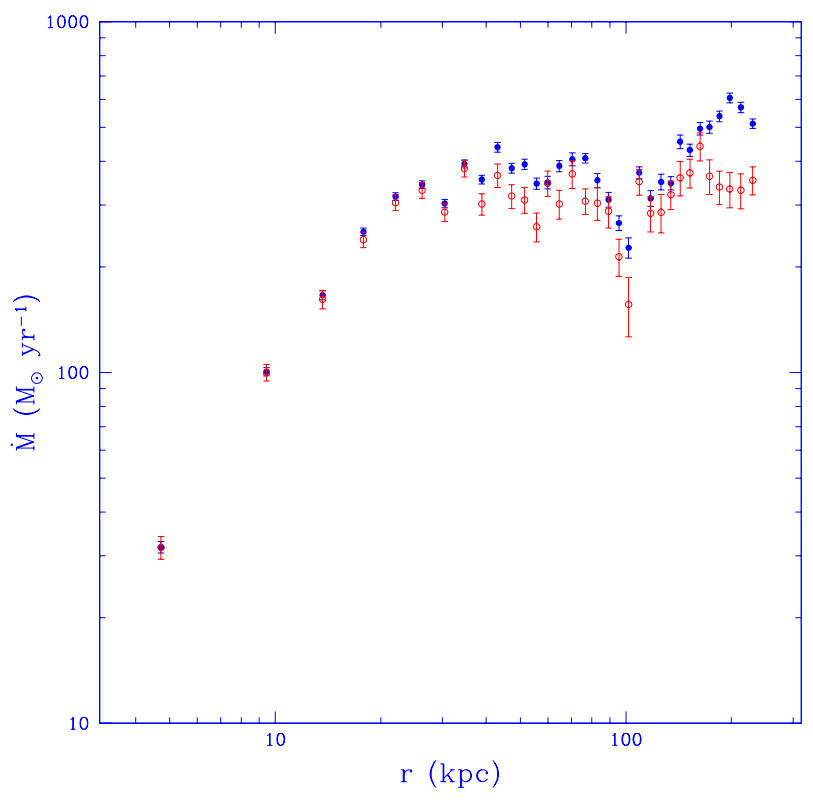

FIG. 17. Morphological $\dot{M}$ profile derived from the deprojected surface brightness and temperature profiles. Filled circles only include the change in gas enthalpy across a shell, while the open circles include the change in enthalpy and gravitational potential in eq. (4). 